ARTICLE

\title{
Cancer-associated fibroblast heterogeneity in axillary lymph nodes drives metastases in breast cancer through complementary mechanisms
}

\author{
Floriane Pelon (1) 1,2, Brigitte Bourachot (10) 1,2, Yann Kieffer (10) 1,2, llaria Magagna (1) 1,2, Fanny Mermet-Meillon (10 ${ }^{3}$, \\ Isabelle Bonnet (1) 4, Ana Costa (1) 1,2, Anne-Marie Givel 1,2, Youmna Attieh (1) 5, Jorge Barbazan (1D) ${ }^{5}$, \\ Claire Bonneau (10) ${ }^{1,2}$, Laetitia Fuhrmann (10) ${ }^{6}$, Stéphanie Descroix (iD) ${ }^{7}$, Danijela Vignjevic (10) ${ }^{5}$, Pascal Silberzan (10 ${ }^{4}$, \\ Maria Carla Parrini (iD ${ }^{3}$, Anne Vincent-Salomon ${ }^{6}$ \& Fatima Mechta-Grigoriou (i) 1,2*
}

\begin{abstract}
Although fibroblast heterogeneity is recognized in primary tumors, both its characterization in and its impact on metastases remain unknown. Here, combining flow cytometry, immunohistochemistry and RNA-sequencing on breast cancer samples, we identify four CancerAssociated Fibroblast (CAF) subpopulations in metastatic lymph nodes (LN). Two myofibroblastic subsets, CAF-S1 and CAF-S4, accumulate in LN and correlate with cancer cell invasion. By developing functional assays on primary cultures, we demonstrate that these subsets promote metastasis through distinct functions. While CAF-S1 stimulate cancer cell migration and initiate an epithelial-to-mesenchymal transition through CXCL12 and TGF $\beta$ pathways, highly contractile CAF-S4 induce cancer cell invasion in 3-dimensions via $\mathrm{NOTCH}$ signaling. Patients with high levels of CAFs, particularly CAF-S4, in LN at diagnosis are prone to develop late distant metastases. Our findings suggest that CAF subset accumulation in LN is a prognostic marker, suggesting that CAF subsets could be examined in axillary LN at diagnosis.
\end{abstract}

\footnotetext{
${ }^{1}$ Institut Curie, Stress and Cancer Laboratory, Equipe labélisée par la Ligue Nationale contre le Cancer, PSL Research University, 26, rue d'Ulm, F-75005 Paris, France. ${ }^{2}$ Inserm, U830, 26, rue d'Ulm, F-75005 Paris, France. ${ }^{3}$ Analysis of Transduction Pathway, Institut Curie, Inserm, U830, PSL Research University, 26 rue d'Ulm, F-75005 Paris, France. ${ }^{4}$ Institut Curie, Biology-inspired Physics at MesoScales Laboratory, Equipe labélisée par la Ligue Nationale contre le Cancer, CNRS UMR168, PSL Research University, Sorbonne Université, 26, rue d'Ulm, F-75005 Paris, France. ${ }^{5}$ Institut Curie, Cell Migration and Invasion, UMR144, PSL Research University, 26, rue d'Ulm, F-75005 Paris, France. ${ }^{6}$ Department of Pathology, Institut Curie Hospital, 26 , rue d'Ulm, F-75248 Paris, France. ${ }^{7}$ Institut Curie, Laboratoire Physico Chimie Curie, Institut Pierre-Gilles de Gennes, CNRS UMR168, 75005 Paris, France. *email: fatima.mechtagrigoriou@curie.fr
} 
$\mathrm{B}$ reast cancers (BCs) are the most frequent cancers in women worldwide. Diagnosis is mainly based on molecular subtype (Luminal A/B, HER2 and triple-negative (TN) defined according to cancer cell expression of progesterone, estrogen and HER2 receptors), tumor size and grade, as well as axillary lymph node (LN) metastasis. Most BC deaths arise from distant metastases in bone, liver and lung ${ }^{1,2}$. LNs are first reached by tumor cells evading from primary tumors (PTs) and number of invaded LNs is a strong prognostic factor ${ }^{3}$. Tumor metastasis is a multi-step process, including local invasion, intravasation, migration in blood or lymph stream, extravasation and distant organ colonization. Among others, one mechanism involves cancer cell epithelial-to-mesenchymal transition (EMT), which involves TGF $\beta$ but also NOTCH and WNT signaling pathways $^{4-7}$.

Tumors are complex ecologies composed of numerous cell types, which participate in tumorigenesis and modulate cancer cell invasiveness ${ }^{8-11}$. Cancer-associated fibroblasts (CAFs) are abundant and involved in many tumor hallmarks such as angiogenesis, tumor cell proliferation, treatment resistance, immunomodulation and metastases $9,12-15$. In particular, CAFs are well-described to enhance tumor invasion and metastases, especially in BC. Indeed, CAFs are able to secrete factors, such as TGF $\beta$ and CXCL12, that directly stimulate cancer cell proliferation, EMT and migration ${ }^{16-19}$. Moreover, CAFs indirectly promote tumor spread via angiogenesis induction through VEGF and IL69,20. Besides, CAFs can directly interact with cancer cells via heterotypic E-cadherin-N-cadherin (CDH1-CDH2) adhesion and drive their invasion ${ }^{21}$. They remodel the extracellular matrix $(\mathrm{ECM})^{22,23}$ and produce spaces or even tracks followed by cancer cells ${ }^{24-26}$. Recently, lung epithelial cells were shown to acquire cancer-associated parenchymal-like feature in metastatic niche ${ }^{7}$.

CAFs are poorly described in metastases, including axillary LN metastases, with only few studies assessing some markers, such as podoplanin (PDPN) or $\alpha$-smooth muscle actin (aSMA) ${ }^{27-30}$. CAFs are known to be heterogeneous in $\mathrm{PTs}^{31-34}$, but characterization of this heterogeneity and its link with CAF functions is still far from being understood. If the role of CAFs in tumor invasion is well established, here we investigated if and how CAF subsets act on metastatic spread. As we recently identified four CAF subsets (named CAF-S1 to -S4) in $\mathrm{BC}^{32}$, we investigated CAF heterogeneity in metastatic $\mathrm{LN}$ and tested if one or several of these CAF subsets could be involved in BC cell spread. We show here that two particular subsets, CAF-S1 and CAF-S4, strikingly accumulate in metastatic $\mathrm{LN}$ and positively modulate BC cell invasion by complementary mechanisms. On the one hand, CAFS1 induce cancer cell migration and EMT initiation in a CXCL12/ TGF $\beta$-dependent manner. On the other hand, CAF-S4 contractile properties promote cancer cell motility and invasiveness in 3dimensions (3D) through NOTCH-mediated pathways. Importantly, CAF-S1 and CAF-S4 content in LN is an independent prognostic factor at diagnosis, underlining the clinical relevance of our findings.

\section{Results}

Metastatic breast cancer axillary LNs exhibit four CAF subsets. To investigate CAF heterogeneity in BC metastatic axillary LNs, we first performed multicolor flow cytometry (fluorescence-activated cell sorting (FACS)) on freshly resected invaded LN, with their matched PTs as controls (Supplementary Table 1). We applied the same gating strategy on both tissues by first excluding debris, dead cells, doublets, hematopoietic (CD45+), epithelial $(\mathrm{EPCAM}+)$, endothelial $(\mathrm{CD} 31+)$ and red blood cells $(\mathrm{CD} 235 \mathrm{a}+)$ (Supplementary Fig. 1a). We then characterized the CD45EPCAM - CD31 - CD235a - fraction enriched in fibroblasts by studying five CAF markers: FAP (fibroblast activation protein $\alpha 1$ ), CD29 (Integrin $\beta 1$ ), aSMA, PDGFR $\beta$ (platelet-derived growth factor receptor- $\beta$ ) and PDPN (Fig. 1a). On FACS data, we applied FlowSom ${ }^{35}$, an unsupervised automated algorithm which orders cells according to their phenotypic similarities (Fig. 1b, c). FlowSom clustered CAFs into four main branches and thus distinguished four CAF subsets (CAF-S1 to -S4) in invaded LNs (Fig. $1 \mathrm{~b}$ and Supplementary Fig. 1b). Four branches were also detected in corresponding PT (Fig. 1c and Supplementary Fig. 1c), confirming data in breast and ovarian $\mathrm{PT}^{32,33}$. Although expression of CD29 in CAF-S3 was slightly lower and PDGFR $\beta$ in CAF-S4 slightly higher in LNs than in PTs, systematic pairwise comparison of each CAF marker protein level between LNs and PTs showed the identification of CAF-S1, -S2, -S3 and -S4 in metastatic LNs (Fig. 1d, e). Hence, these data reveal that four CAF subsets are detected in metastatic BC LNs.

As normal LN structure relies on a fibroblastic network constituted by fibroblast reticular cells (FRCs) described as $\mathrm{PDPN}+$ cells $^{36}$, we investigated the analogy between normal stromal cells and CAF subsets in LNs. Even though non-invaded LNs were hardly accessible because almost fully used for diagnosis, we had access to two non-invaded specimens (Supplementary Fig. 1d), along with their matched invaded LNs. Non-invaded axillary LNs were clearly enriched in CAF-S2- and CAF-S3-like cells, while the matched invaded LNs showed a much higher proportion of CAF-S1 and CAF-S4 (Fig. If and Supplementary Fig. 1e). CAF-S2 and CAF-S3 subpopulations are thus detected in metastatic LNs, but also in non-invaded LNs. These results corroborated our previous data showing that CAF-S2- and CAFS3-like cells are detected in healthy breast tissue ${ }^{32}$, suggesting that these CAFs might derive from normal resident fibroblasts. In that sense, the pattern of CAF-S3 in LNs was slightly different than the one detected in PTs, as observed with CD29 staining (Fig. 1b-d), suggesting that normal-like CAF-S2/S3 could be more affected by their tissue of origin than CAF-S1 and CAF-S4. In contrast to CAF-S2 and CAF-S3, CAF-S1 and CAF-S4 were strictly observed in invaded LNs and positively correlated with tumor cell invasion (Fig. 1g). Thus, these data highlight a potential link between both CAF-S1 and CAF-S4 and tumor cell invasion in LNs. In conclusion, we identified four CAF subsets in metastatic LNs defined as: CAF-S1: FAPHigh CD29Med-High aSMA ${ }^{\text {High }}$ PDPN $^{\text {High }}$ PDGFR $\beta^{\text {High }}$; CAF-S2: FAPNeg CD29 ${ }^{\text {Low }} \alpha$ SMA ${ }^{\text {Neg-Low }}$ PDPN ${ }^{\text {Low }}$ PDGFR $\beta^{\text {Low; }}$ CAF-S3: FAPNeg-Low CD29Med aSMA ${ }^{\text {Neg-Low }}$

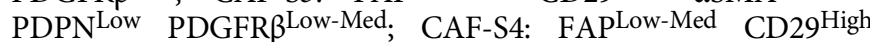
aSMA ${ }^{\text {High }}$ PDPN ${ }^{\text {Low }}$ PDGFR $\beta^{\text {Med }}$. Besides, the amounts of both CAF-S1 and CAF-S4 subsets in LNs are linked to BC cell metastatic spread.

CAF-S1 and CAF-S4 are the most abundant subsets in metastatic LN. To decipher the link between CAF subsets and metastatic spread, we studied metastatic LN sections from a retrospective cohort of $124 \mathrm{BC}$ patients (Supplementary Table 2). We analyzed invaded zones of metastatic LN, identified using EPCAM marker (Supplementary Fig. 2a). We first observed that LN stroma represented around $25-30 \%$ of invaded areas, independently of BC subtypes (Fig. 2a). We performed immunohistochemistry (IHC) of five CAF markers (FAP, CD29, FSP1, PDGFR $\beta$, aSMA) on serial LN sections (Fig. $2 b, c)$. Here, we replaced PDPN by FSP1 because we could not find a PDPNspecific antibody for IHC, but we verified that PDPN and FSP1 markers recognized the same cells by FACS (Supplementary Fig. 2b). Histological scoring of each CAF marker demonstrated that invaded LNs from Luminal (Lum A and B) cases exhibited the lowest histological scores (H-scores) except for PDGFR $\beta$, whereas both HER2 and TN LNs showed the highest H-scores 
a
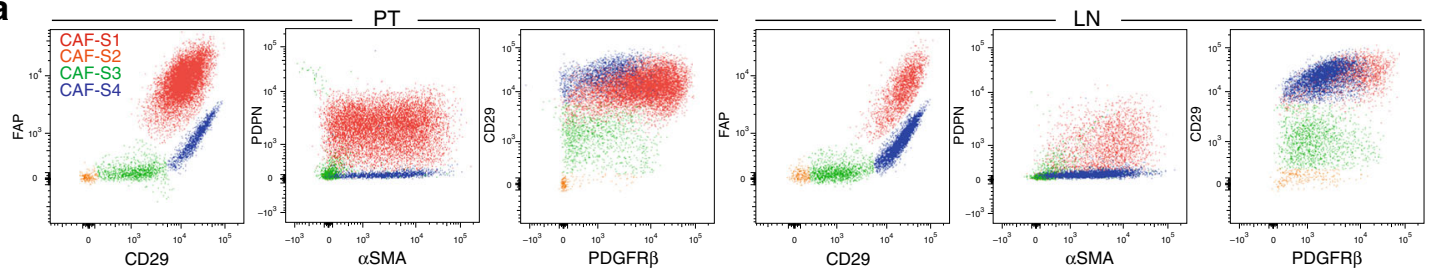

b

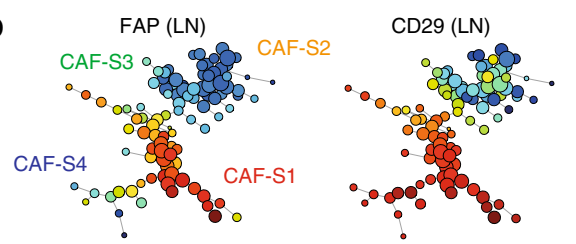

C
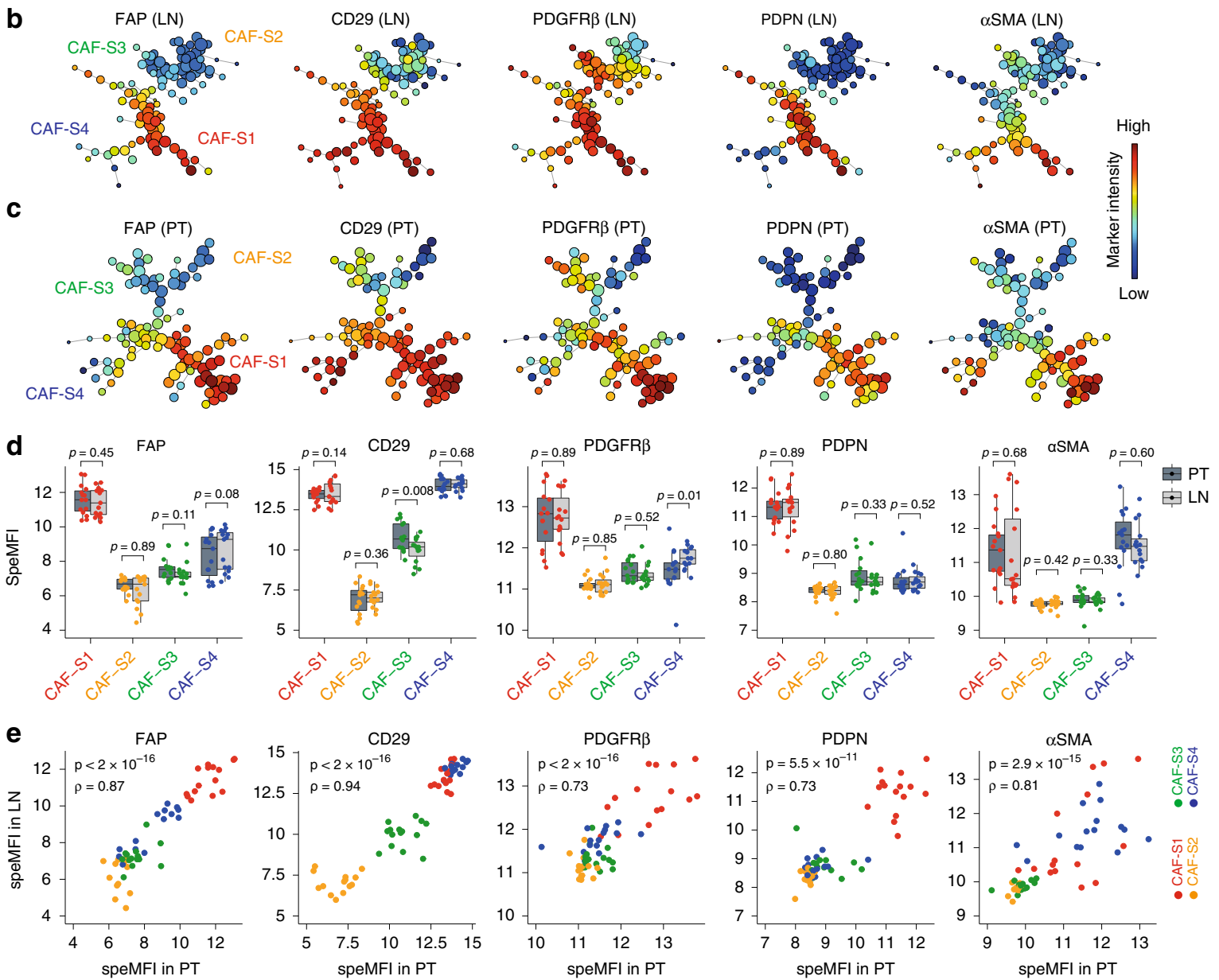

$\mathbf{f}$
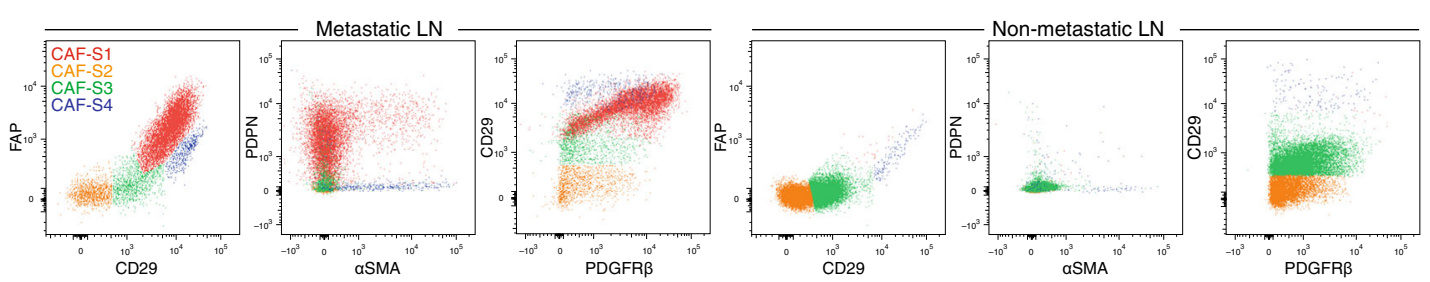

g
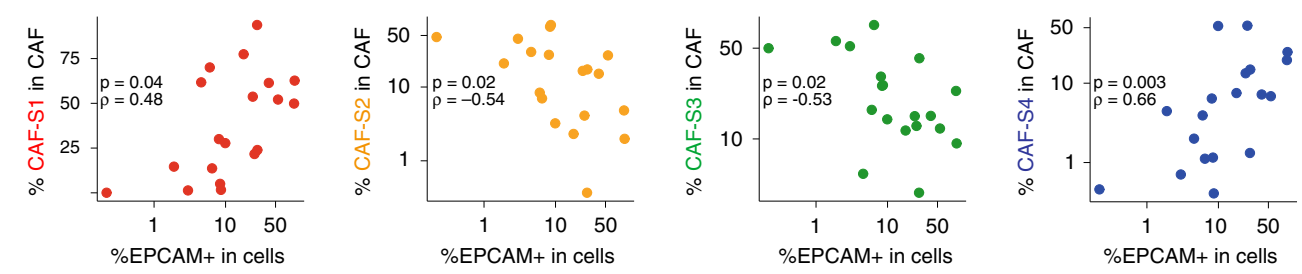

(Fig. 2b, c and Supplementary Fig. 2c). When applying a decision tree algorithm to determine CAF subset enrichment ${ }^{32}$ (Fig. 2d), we found that $96 \%$ of metastatic LNs showed accumulation of CAF-S1 and CAF-S4 (Fig. 2e). Luminal LNs were mainly enriched in CAF-S4, while HER2 and TN cases displayed both CAF$\mathrm{S} 1$ and CAF-S4 predominance. We observed that the median

percentage of fibroblasts positive for FAP, SMA and CD29 (reflecting CAF-S1 identity) reached $75 \%$ of total CAFs in CAFS1-enriched LNs, and that fibroblasts negative for FAP but positive for SMA and CD29 (reflecting CAF-S4 identity) reached $60 \%$ of total fibroblasts in CAF-S4-enriched LNs. We also developed an image analysis tool that combined spatial 
Fig. 1 Metastatic BC LNs exhibit four CAF subsets. a Representative FACS plots showing FAP, CD29, PDPN, $\alpha$ SMA and PDGFR $\beta$ protein levels in DAPIEPCAM $^{-} \mathrm{CD}^{-} 5^{-} \mathrm{CD}^{-} 1^{-} \mathrm{CD} 235 \mathrm{a}^{-}$cells from a primary breast tumor (PT, left) and its corresponding metastatic axillary lymph node (LN, right). b, c FlowSom trees built on CAF from LNs $(\mathbf{b}, n=20)$ and PTs $(\mathbf{c}, n=16)$ and annotated for each CAF marker expression. Colors show CAF marker intensities. Node size depends on the number of phenotypically close cells. d Specific mean fluorescence intensity (speMFI) for each marker per CAF subset in PT and LN. Values are in log2 adjusted with offsets per marker. Each dot represents one sample ( $n \geq 13$ PT/LN pairs). Boxplots are median $\pm 25 \%-75 \%$ quantiles, whisker values range $1.5 \times I Q R$ above 75 th or below 25th percentiles. $p$ Values from Wilcoxon signed rank test. e Correlation plots between each marker speMFI in PT and LN, matched by patient and CAF subset ( $n \geq 13$ PT/LN pairs). $p$ Values from Spearman's test. f Same as in a for an invaded axillary LN (left) and its corresponding non-invaded LN (right). $\mathbf{g}$ Correlation plots between the percentage (\%) of each CAF subset among total CAF and EPCAM ${ }^{+}$ cells among live cells, in invaded axillary LN $(n=19)$. $p$ Values from Spearman's test. Source data provided in Source Data file, with R scripts used.

registration and computational analysis to generate maps of CAF subsets at cellular level in invaded LNs (Fig. $2 \mathrm{f}$ and Supplementary Fig. 2d). Representative pictures of CAF subset maps confirmed the enrichment in CAF-S1 or CAF-S4 in invaded LNs. Interestingly, among the 124 LNs analyzed, we had access to 41 corresponding PTs (Lum A, HER2, TN) for matched analyses (Fig. 2g and Supplementary Fig. 2e). We found that FAP and aSMA H-scores were similar between PTs and LNs, whereas CD29 and PDGFR $\beta$ were higher and FSP1 lower in LNs (Supplementary Fig. 2e). When we combined H-scores using the decision tree algorithm, we found that CAF enrichments in metastatic LNs were significantly different from those in matched PTs, with a strong increase in CAF-S4 in LNs (Fig. 2g). Similarly, when comparing invaded LNs to PTs (unmatched samples) from a larger BC cohort with at least one invaded $\mathrm{LN}$ at diagnosis $(\mathrm{N}+)$ (Supplementary Table 2), we confirmed the accumulation of CAF-S4, the increase in CAF-S1 and the decrease in CAF-S2 and CAF-S3 in invaded LNs compared to PTs (Fig. 2h). Metastatic LNs were particularly enriched in CAF-S4 compared to PTs in Luminal A and TN subtypes (Fig. 2i), possibly because HER2 PTs exhibited already high CAF-S4 content. As CAF marker H-scores were significantly correlated between PTs and LNs (FAP: $r=$ 0.54, $p=3.10-4$; CD29: $r=0.45, p=3.10-3$; FSP1: $r=0.52, p=$ 6.10-4; SMA: $r=0.44, p=4.10-3$ by Pearson's correlation test), we hypothesized that CAF-S1-enriched tumors could give rise to CAF-S1-enriched LNs, with similar consideration for CAF-S4. Indeed, 79\% of CAF-S1-enriched PTs matched with CAF-S1enriched LNs and 71\% of CAF-S4-enriched PTs matched with CAF-S4-enriched LNs (Fig. 2j) ( $p=0.007$ by Fisher's exact test). Reciprocally, CAF-S1-enriched LNs mostly matched to CAF-S1enriched PTs (69\%). In contrast, CAF-S4-enriched LNs corresponded to PTs enriched in different CAF subsets $(p=0.005$ by Fisher's exact test), suggesting that CAF-S4 in LNs could derive from distinct CAF subsets. In conclusion, our data show that metastatic LN stroma is enriched in myofibroblastic CAF-S1 and CAF-S4 subsets. Interestingly, CAF enrichments are different in LNs compared to PTs since LN accumulating CAF-S4 can correspond to different types of $\mathrm{PT}$ s that are enriched either in CAF$\mathrm{S} 4$ or in inactivated CAFs (CAF-S2 or CAF-S3).

CAF subsets show similar signatures between PTs and metastatic LNs. As CAF-S1 and CAF-S4 subsets were largely predominant in LNs, we next aimed at characterizing these LN CAF subsets on a molecular basis and compared them to PT CAF subsets. CAF-S1 and CAF-S4 from both PTs and LNs $(n=5$ pairs) were FACS-sorted by excluding dead, hematopoietic, epithelial, endothelial cells and erythrocytes and analyzing CAF markers (using the same gating strategy as in Supplementary Fig. 1). We performed RNA sequencing (RNAseq) on CAF-S1 and CAF-S4 fibroblasts, and EPCAM+ cancer cells sorted from PTs and matched invaded LNs (Supplementary Table 1). Unsupervised principal component analysis (PCA) and hierarchical clustering (HC) built on the 500 most variant genes distinguished tumor cells, CAF-S1 and CAF-S4 fibroblasts (Fig. 3a, b). The first
PCA component (51\% variance) differentiated EPCAM+ cells from CAF, and the second (23\% variance) CAF-S1 from CAF-S4 (Fig. 3a). HC enabled us to visualize specific transcriptomic profiles (Fig. 3b). PCA and HC showed that samples from PTs and LNs were mixed within each cellular population, highlighting that differences between CAF subsets (CAF-S1/CAF-S4) were higher than between tissue of origin $(\mathrm{PT} / \mathrm{LN})$. We performed PCA on each population on their respective 500 most variant genes and still noted that PT and LN samples did not segregate (Fig. 3c). In line with published data 4,37 , tumor cells clustered with their matched EPCAM + LN cells, suggesting that tumor cell transcriptomes vary more between patients than between PTs and LNs. In contrast, patient effect was less detectable in CAF-S1 or CAF-S4 (Fig. 3c). We next performed paired differential analyses of CAF-S1 and CAF-S4 considering either PT or LN samples. CAF-S1 upregulated genes were mainly involved in ECM organization and CAF-S4-specific genes in muscle contraction (Supplementary Table 3 and Fig. 3d). As CAF-S4 exhibited pericyte-like signature, we confirmed that we could detect MCAM-positive fibroblasts in invaded LNs (Supplementary Fig. 2f), thereby confirming the existence of pericyte-like CAF and their potential pericyte origin ${ }^{32,38-41}$. Moreover, there was a strong overlap between $\mathrm{PT}$ and $\mathrm{LN}$ signatures for each CAF subset (Fig. 3d), suggesting that CAF-S1 and CAF-S4 from metastatic LN are molecularly close to CAF-S1 and CAF-S4 from PTs (Supplementary Tables 4 and 5).

CAF-S1 and CAF-S4 exhibit distinct migration and invasion properties. As invaded LNs show predominantly CAF-S1 and CAF-S4 fibroblasts, we hypothesized they could be involved in metastatic spread and we analyzed their properties by functional assays. We established CAF-S1 and CAF-S4 primary cell cultures from BC patients. We first confirmed by RNAseq on four CAF-S1 and CAF-S4 pairs (three couples from PTs and one from LN) that, after several passages in culture, these cells kept similar molecular identities as those detected without culture (Supplementary Fig. 3a-c). Moreover, we confirmed by FACS that CAF markers at protein levels corroborated data from fresh samples (Supplementary Fig. 3d). We next deciphered CAF-S1 and CAFS4 intrinsic characteristics. We first observed that CAF-S1 proliferated slightly faster than CAF-S4 (median doubling time = 2.1 days for CAF-S1 and 3.0 days for CAF-S4) (Fig. 4a). Moreover, CAF-S1 displayed higher migration skills compared to CAF-S4, as assessed by Transwell assays (Fig. 4b). We validated this result using cell exclusion zone assays by manually tracking cells and calculated their velocity, persistence and directionality. CAF-S1 were faster and more persistent than CAF-S4, CAF-S1 trajectories being more perpendicular to the edges than CAF-S4 (Fig. 4c and Supplementary Fig. 3e). We also analyzed CAF subset invasion properties in 3D. Both CAF-S1 and CAF-S4 subsets were able to form spheroids in hanging drops and to invade the surrounding matrix but CAF-S1 were more efficient in invading than CAF-S4 (Fig. 4d). Interestingly, on time-lapse video microscopy (Supplementary Movies), we observed that 
a

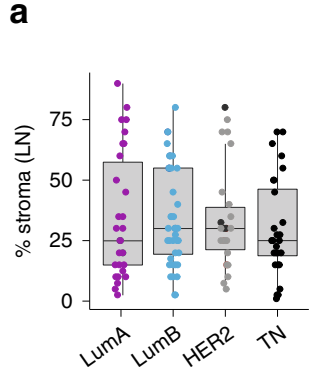

C

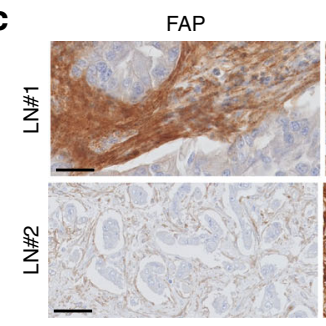

d

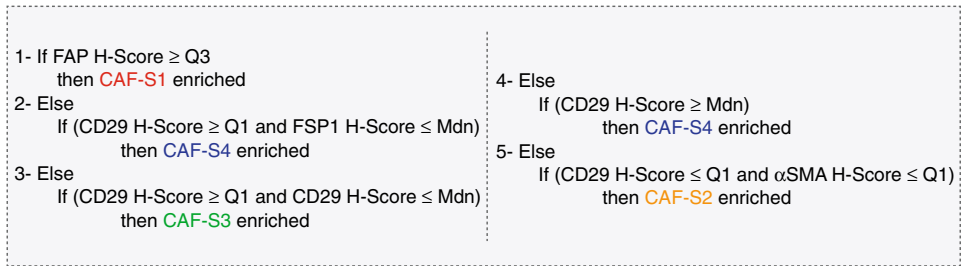

b

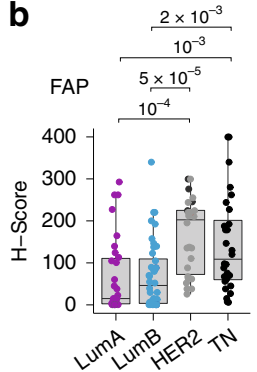

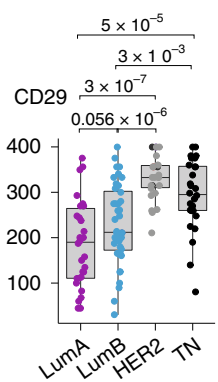

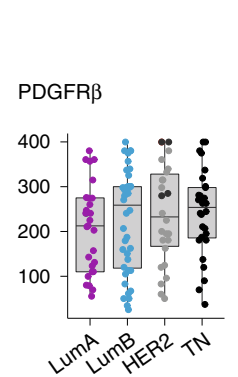

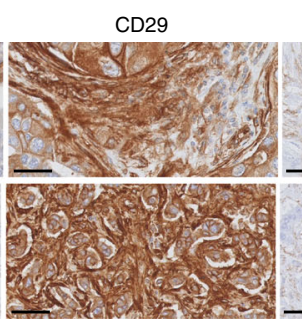

PDGFR $\beta$
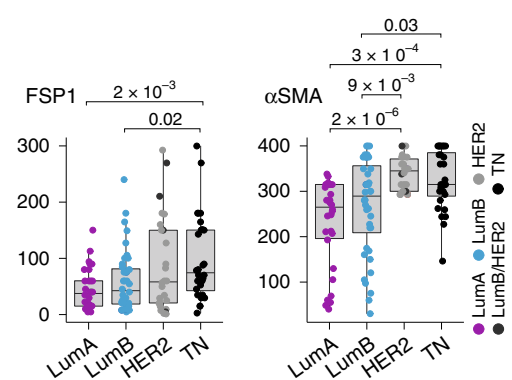
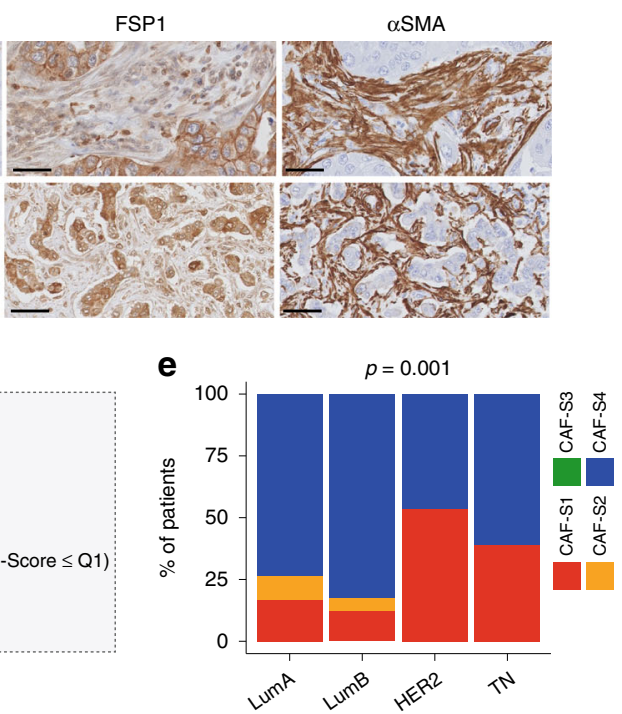

f
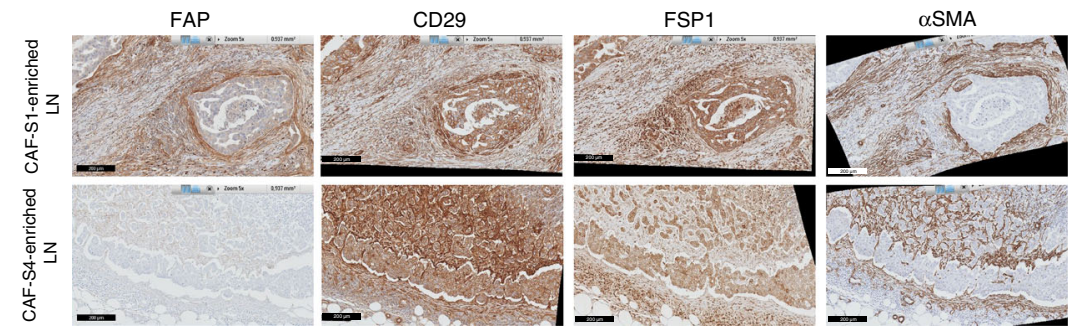

Map at cellular scale
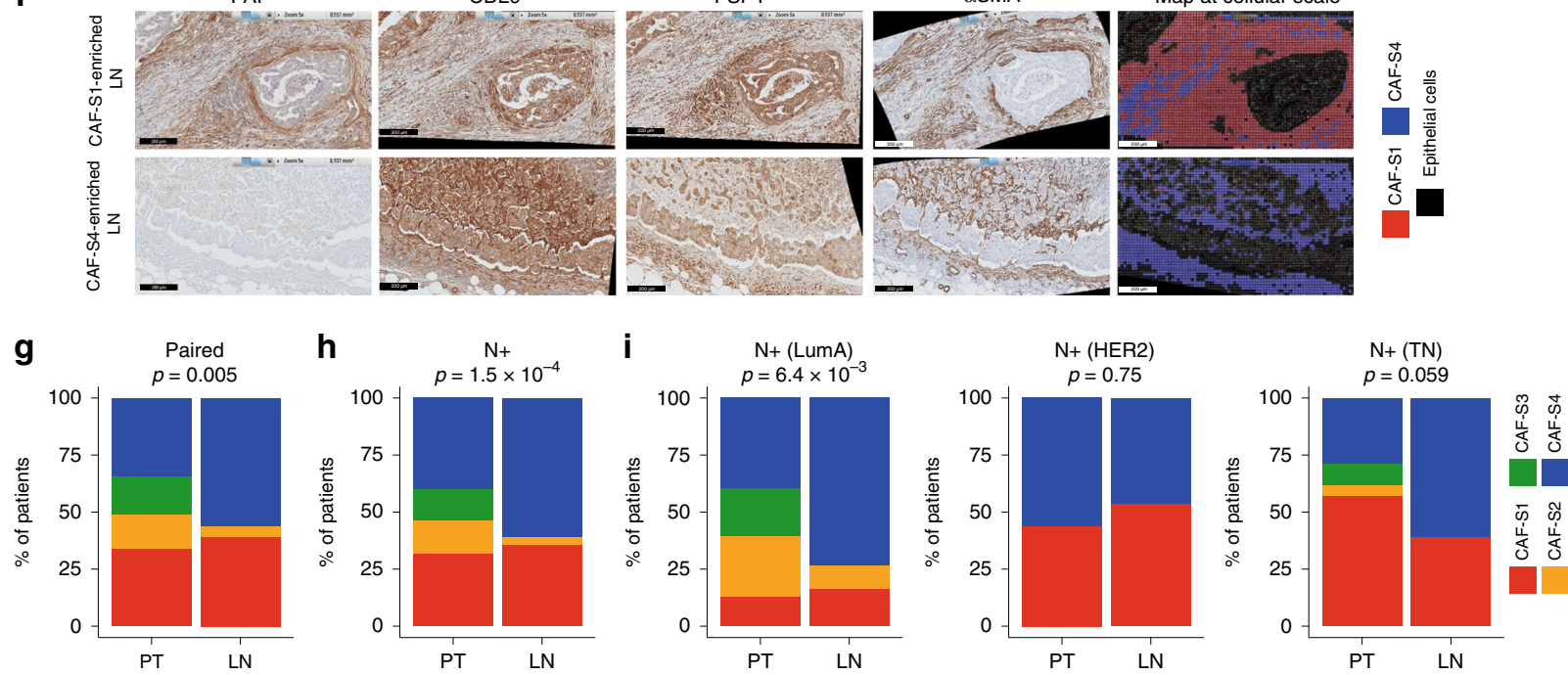

\begin{tabular}{|c|c|c|c|c|c|}
\hline & PT-CAF-S1 & PT-CAF-S2 & PT-CAF-S3 & PT-CAF-S4 & TOTAL \\
\hline LN-CAF-S1 & 11 & 1 & 1 & 3 & 16 \\
\hline LN-CAF-S2 & 0 & 1 & 0 & 1 & 2 \\
\hline LN-CAF-S3 & 0 & 0 & 0 & 0 & 0 \\
\hline LN-CAF-S4 & 3 & 4 & 6 & 10 & 23 \\
\hline TOTAL & 14 & 6 & 7 & 14 & 41 \\
\hline
\end{tabular}

spheroids made by CAF-S4 pulled more efficiently on collagen fibers than CAF-S1 (Fig. 4e). To further quantify the traction forces exerted by CAF on matrix, we performed traction force microscopy (TFM). We observed that CAF-S1 were more elongated compared to CAF-S4 that were larger (Fig. 4f and
Supplementary Fig. 3f, h). Moreover, the strain energy (that measures the energy spent to deform the substrate) developed by CAF-S4 was more than twofold higher than that of CAF-S1 (Supplementary Fig. 3g). To take into account differences in surface area between CAF-S1 and CAF-S4 (Supplementary 
Fig. 2 Metastatic LNs are enriched in CAF-S1 and CAF-S4. a Percentage (\%) of stroma in invaded LN according to BC subtypes ( $n=124)$, LumA (violet), LumB (blue), HER2 (light gray), Lum B/HER2 (dark gray), TN (black). b Histological scores (H-Scores) for each CAF marker in invaded LN according to BC subtypes $(n=124)$. c Representative images of CAF marker staining on serial LN sections (LN\#1 HER2; LN\#2 TN). Scale bar, $50 \mu$ m. $\mathbf{d}$ Decision tree defining CAF subset identity based on quartile $(\mathrm{Q})$ and median (Mdn) distribution of CAF markers. Thresholds and decision rules were first established on FACS data and next applied to IHC data. e Repartition of CAF subset enrichments (CAF-S1 (red), CAF-S2 (orange), CAF-S3 (green) and CAF-S4 (blue)) in $\mathrm{LN}$ according to $\mathrm{BC}$ subtypes $(n=124)$. $\mathbf{f}$ Representative views of CAF marker immunostaining on serial $L N$ sections used for building maps of CAF subsets at cellular scale using the decision tree algorithm, shown in d. CAF-S1 are in red, CAF-S4 in blue and epithelial cells in black. CAF-S1- and CAF-S4-enriched LNs are shown. Scale bar, $200 \mu \mathrm{m}$. $\mathbf{g}$ Repartition of CAF subset enrichments in PTs and matched LNs $(n=41$ pairs). $\mathbf{h}$ Same as in $\mathbf{g}$ with unmatched samples ( $\mathrm{N}+$ cases, $n=75$ PT and $84 \mathrm{LN}$ ). i Same as in $\mathbf{h}$ according to BC subtypes (Lum A: $n=38$ PTs, $30 \mathrm{LNs}$; HER2: $n=16$ PTs, 26 LNs; TN: $n=21$ PTs, $28 \mathrm{LNs}$ ). $\mathbf{j}$ Contingency table showing repartition of CAF subset enrichments in PTs and corresponding invaded LNs ( $n=41$ pairs). In all panels, boxplots are median $\pm 25 \%-75 \%$ quantiles, whisker values range $1.5 \times$ IQR above 75 th or below 25th percentiles. b $p$ Values from Mann-Whitney test. e, $\mathbf{g}$, h $p$ Values from Fisher's exact test. Non-significant $p$ values are not mentioned. Source data provided in Source Data file, with $\mathrm{R}$ scripts used.

a

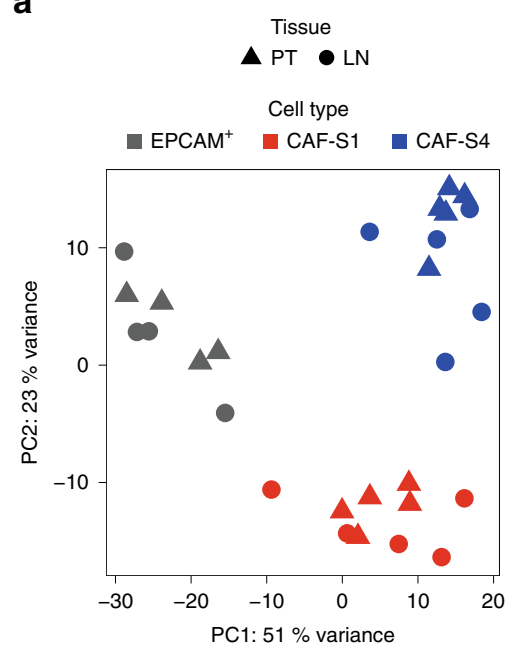

b

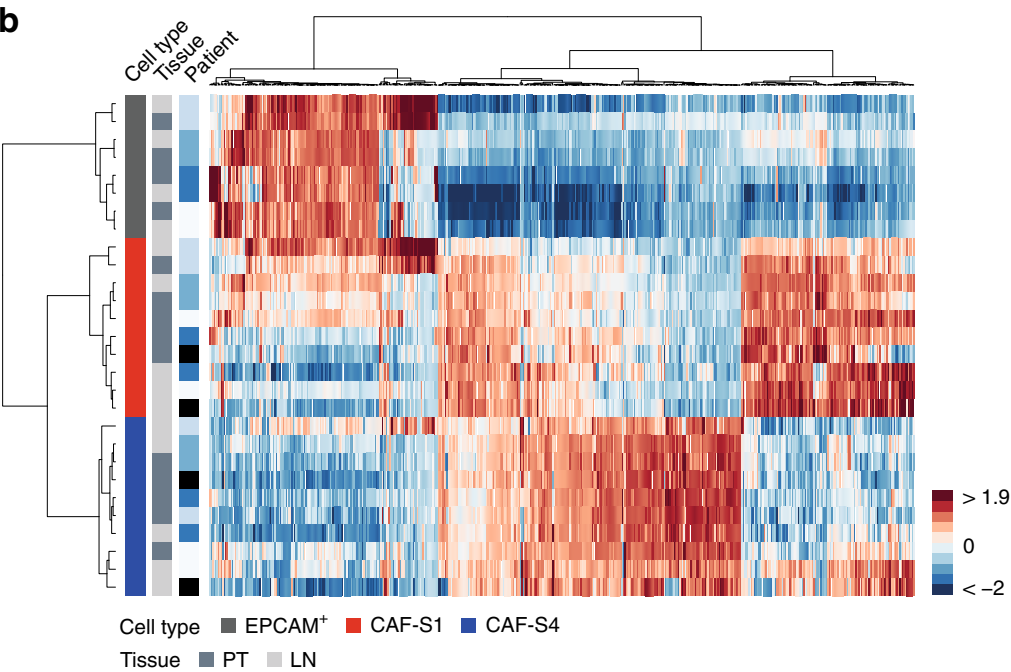

C
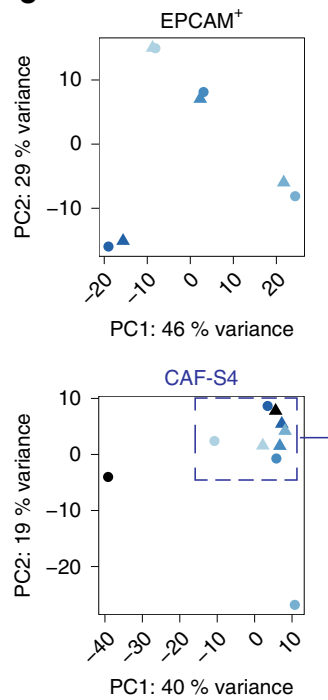
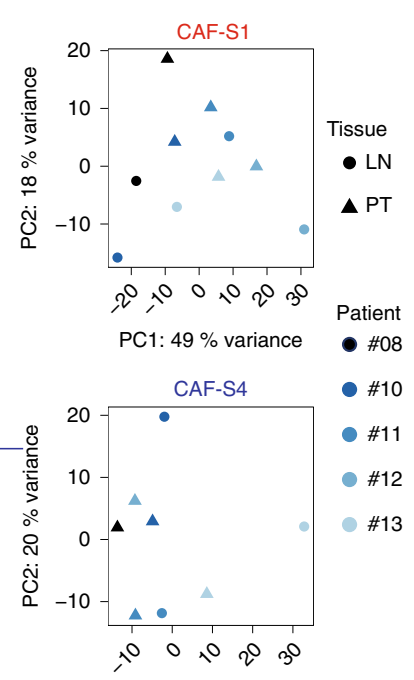

PC1: $39 \%$ variance

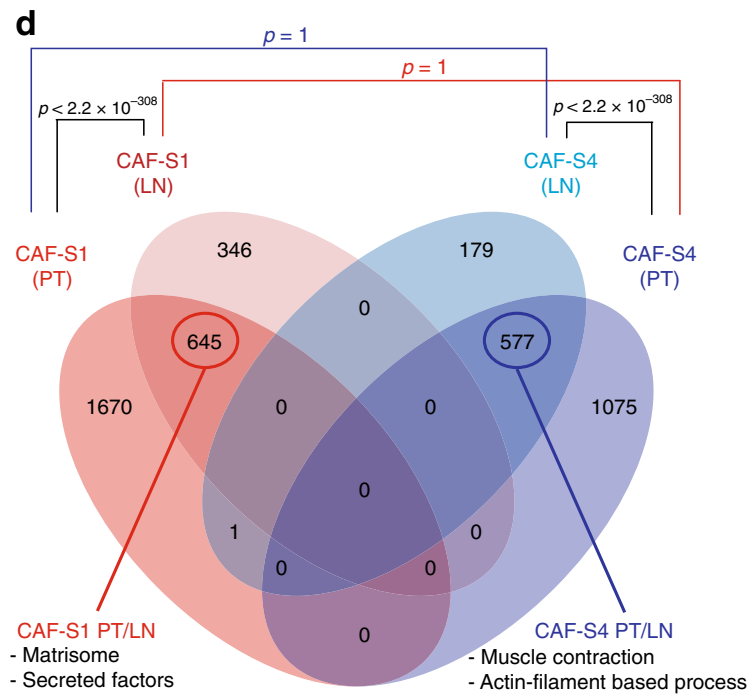

Fig. 3 CAF subsets show same identity in PTs and metastatic LNs. a PCA based on the 500 most variant genes from RNAseq data of CAF-S1, CAF-S4 and EPCAM ${ }^{+}$cells sorted from paired PT and LN ( $n=5$ pairs for CAF-S1 and CAF-S4; $n=4$ pairs for EPCAM ${ }^{+}$cells). b Hierarchical clustering on the same samples and same genes as in a using Ward's method with Pearson distances. Rows represent samples and columns genes. Color saturation shows gene expression deviation from the mean (above in red, below in blue). c PCA based on the 500 most variant genes from RNAseq data of EPCAM ${ }^{+}$cells ( $n=4$ PT/LN pairs, top left), CAF-S1 ( $n=5$ PT/LN pairs, top right) and CAF-S4 (bottom left: $n=5 \mathrm{PT} / \mathrm{LN}$ pairs; bottom right: PCA restricted to $n=8$ samples, as indicated). d Venn diagram showing overlaps between the transcriptomic signatures of CAF-S1 from PT, CAF-S1 from LN, CAF-S4 from PT and CAF-S4 from LN. Circled numbers show common genes between tissue localization for each cell type. $p$ Values from hypergeometric test indicate significance of overlaps between subgroups. Source data provided in Source Data file, with R scripts used. 

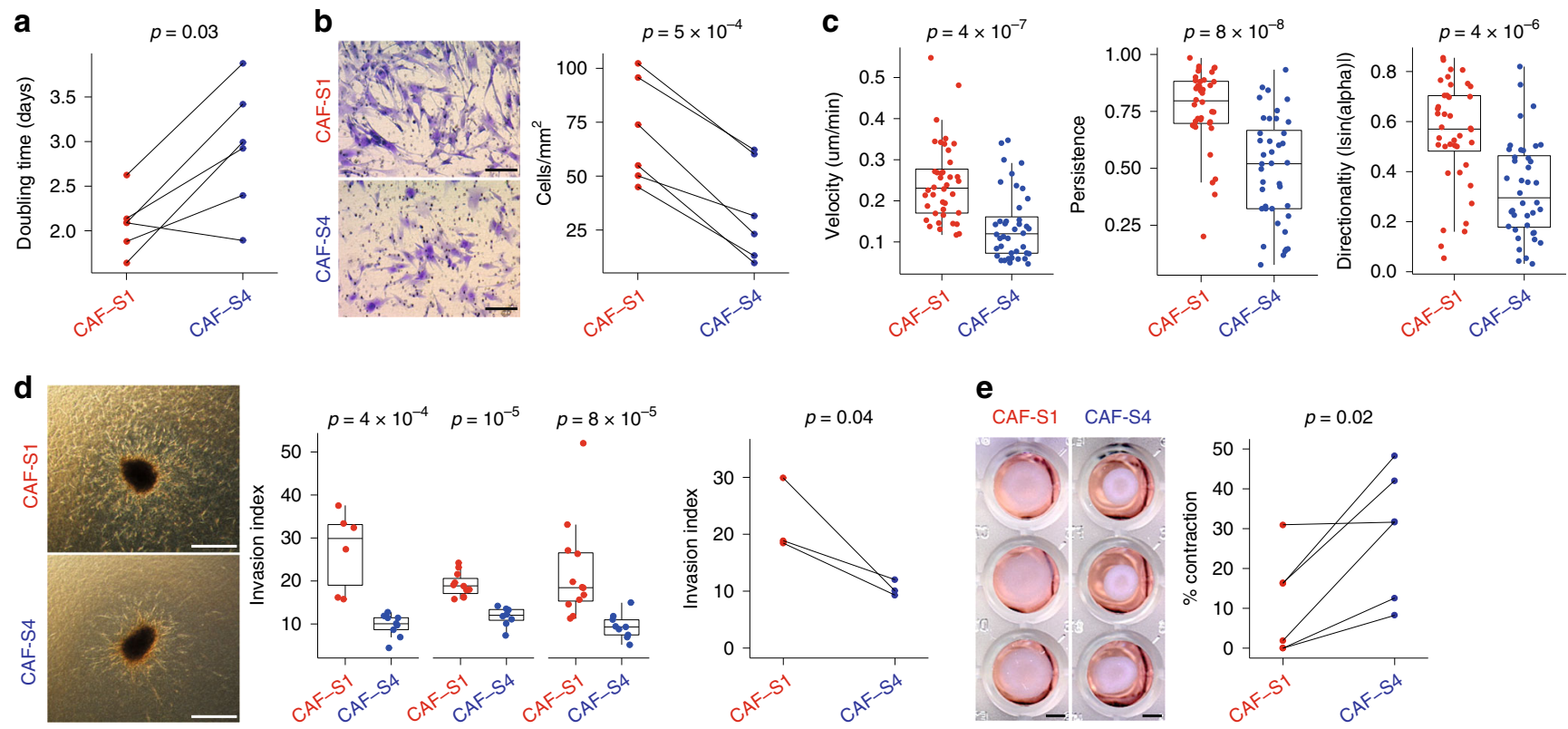

f
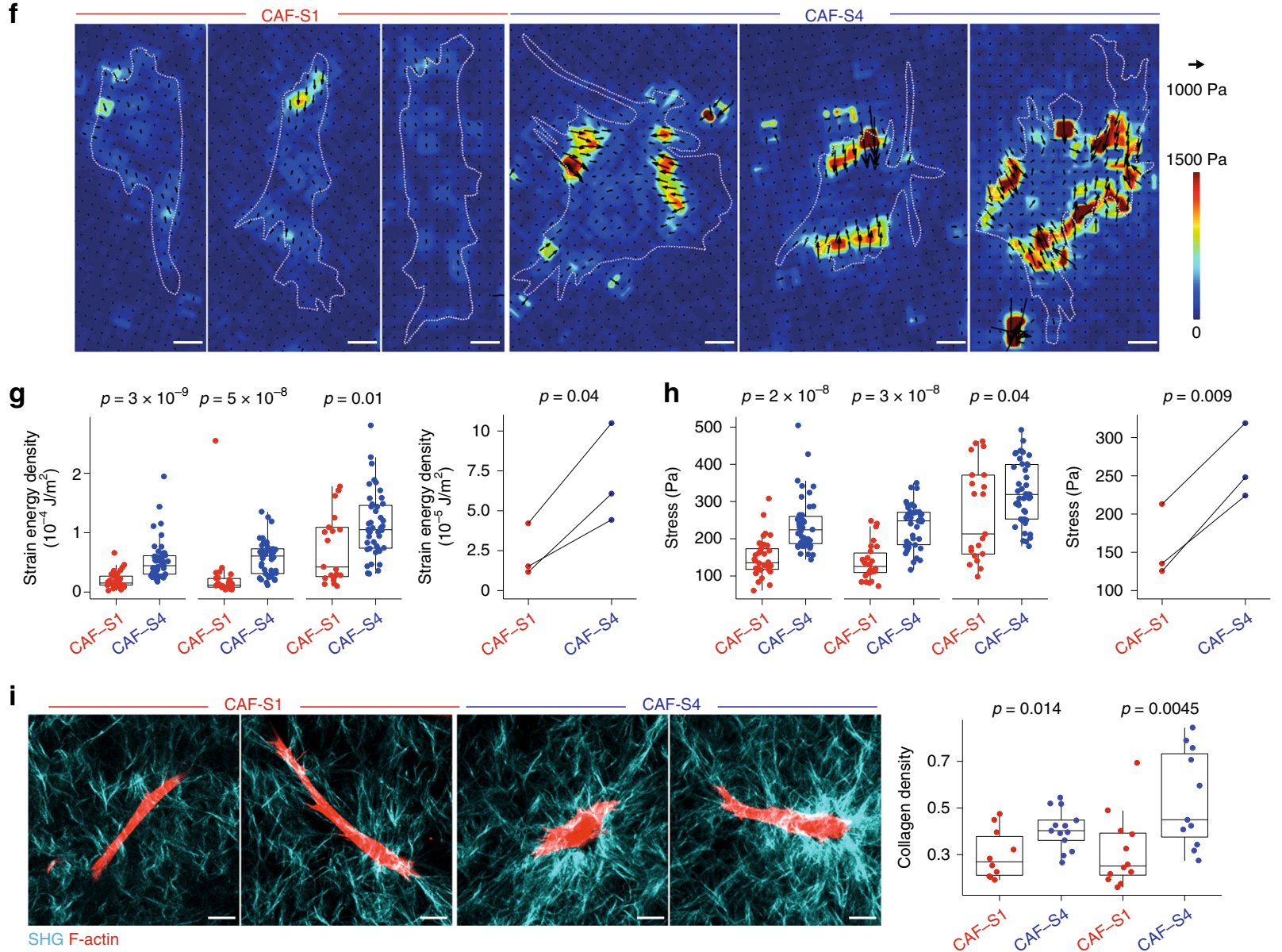

Fig. 3h), we compared the strain energy densities (strain energy normalized to the cell surface) and confirmed that CAF-S4 deployed more contractile energy per surface unit than CAF-S1 (Fig. 4g). Similar results were obtained using mean traction stress amplitude (mechanical forces cell develop per unit area to deform the substrate; arrows Fig. 4f, h). Collagen fiber density was also increased around CAF-S4 compared to CAF-S1 cells (Fig. 4i), which is consistent with CAF-S4 capacity to exert higher traction forces on their environment, to pull and contract collagen fibers. Overall, these data demonstrate that CAF-S1 are motile, while CAF-S4 are contractile with strong capacity to pull on collagen fibers.

CAF-S1 and CAF-S4 promote tumor cell spread by distinct mechanisms. While CAF function in metastases was investigated in the past, the specific role of CAF subsets on metastatic spread 
Fig. 4 CAF-S1 and CAF-S4 exhibit distinct functional features. a CAF-S1 (red) and CAF-S4 (blue) doubling time $(n=6)$. b Left, Transwell membrane underside images. Scale bar, $200 \mu \mathrm{m}$. Right, CAF-S1/-S4 migration capacity (cells/mm²) $(n=6)$. c CAF-S1/-S4 velocity, persistence and direction (|sin $(\alpha) \mid$, assessed by cell exclusion assay. d Left, Images of CAF-S1/-S4 spheroids embedded into collagen. Scale bar, 200 $\mu$ m. Middle, CAF-S1/-S4 invaded areas/core spheroid areas. Each dot is one spheroid ( $n \geq 6$ per CAF subset). Right, Same as Middle for median values per CAF subset. e Left, Images of collagen-gel contraction by CAF-S1/-S4. Scale bar, $2 \mathrm{~mm}$. Right, Percentage of collagen contraction by CAF-S1/-S4 $(n=6)$. $\mathbf{f}-\mathbf{h}$ Contractility of CAF-S1/-S4 by traction force microscopy. $\mathbf{f}$ Images of traction stress applied by CAF-S1/-S4 on substrate. Traction forces (arrows) and cellular outlines (dashed lines) shown. Scale bar, $20 \mu \mathrm{m}$. Traction stress magnitudes in Pascal $(\mathrm{Pa})$. $\mathbf{g}$ Left, CAF-S1/-S4 strain energy density $\left(\mathrm{Joules}(\mathrm{J}) / \mathrm{m}^{2}\right)$. Each dot is one cell $(n \geq$ 21 cells/CAF subset). Right, Same as left for median strain energy densities per CAF subset $(n=3)$. $\mathbf{h}$ Left, CAF-S1/-S4 traction stress. Right, Same as left for median traction stress per CAF subset $(n=3)$. i Left, Images of collagen (blue) by CAF-S1/-S4 (red) assessed by second harmonic generation. Scale bar, $20 \mu \mathrm{m}$. Right, Collagen density in each cell stack. Each dot is the average value of collagen density around one cell ( $n \geq 10$ cells per CAF subset) $(n=2)$. In all panels, boxplots are median $\pm 25 \%-75 \%$ quantiles, whisker values range $1.5 \times 1 \mathrm{IQR}$ above 75 th or below 25 th percentiles. $\mathbf{a}, \mathbf{b}, \mathbf{e}$ right, $\mathbf{g}$ right, $\mathbf{h}$ right: $p$ Value from paired $t$-test. $\mathbf{c}$, $\mathbf{g}$ left: $p$ Values from Mann-Whitney test. $\mathbf{d}$ middle, $\mathbf{h}$ left, $\mathbf{i}$ right for two first CAF pairs: $p$ Values from Mann-Whitney test (1st pair) and Student's t-test (2nd pair). d right: $p$ Value from Student's $t$-test. At least three CAF-S1/-S4 pairs tested, except in c/i, two pairs. Source data provided in Source Data file, with R scripts used.

remains unknown. We assessed CAF-S1 and CAF-S4 effects on BC cell properties by using MCF7 and T47D luminal cell lines, as CAF switch between PTs and LNs was prominent in luminal cases. Co-culture of BC cells with CAF-S1 or CAF-S4 increased the number of tumor cells, with a stronger impact of CAF-S1 compared to CAF-S4 (Fig. 5a). This effect was also detected with CAF-S1 - or CAF-S4-conditioned medium (CM), although at a lower extent (Fig. 5b). CAF subset proliferation increased at long time points of co-culture with cancer cells (Fig. 5c), but not at short term (Supplementary Fig. 4a). Transwell assays showed that $\mathrm{BC}$ cell migration was strongly increased toward CAF-S1, but at a much lower extent toward CAF-S4 (Fig. 5d). The CAF-S1-promigratory effect was independent of its pro-proliferative effect, while the slight impact of CAF-S4 was proliferation-driven (Supplementary Fig. 4b). In agreement with their intrinsic features (Fig. 4), CAF-S4 fibroblasts (easily distinguished from cancer cells by F-actin staining) exhibited more F-actin stress fibers and Vinculin-stained focal adhesions than CAF-S1 (Fig. 5e and Supplementary Fig. 4c for quantification). Interestingly, in presence of CAF-S1 or CAF-S4, cancer cells were more scattered (Fig. 5e and Supplementary Fig. 4c). Indeed, in presence of CAF$\mathrm{S} 1, \mathrm{BC}$ cells were less cohesive and more dispersed. With CAF-S4, cancer cells were also less packed but with numerous remaining F-actin interconnections. In agreement with these observations, E-cadherin/CDH1 protein levels decreased at cancer cell membrane in presence of CAF-S1, effect which was also visible but less pronounced with CAF-S4 (Fig. 5f and Supplementary Fig. 4d). Thus, these results suggest that CAF-S1-and to a lower extent CAF-S4-promote cancer cell spreading by initiating first steps of EMT.

As CAF-S4 properties were linked to $3 \mathrm{D}$ environments, we moved to such systems to analyze their impact on BC cell invasiveness in surrounding matrix. We first used an inverted Transwell assay to test CAF subset-mediated matrix remodeling on BC cell invasion (Fig. 6a-f). In this assay, cancer cells need to cross the membrane to invade the matrix. MCF7 and T47D cells are not invasive enough to obtain quantifiable results, we thus chose to work with MDA-MB-231, a TN invasive cell line. The highest $z$-distance covered by cancer cells in the matrix was significantly increased in CAF-S1- and even more in CAF-S4embedded collagen (Fig. 6a, b). If cancer cells browsed in average $72 \mu \mathrm{m}$ above the membrane in a CAF-free matrix, they reached $109 \mu \mathrm{m}$ in presence of CAF-S1 and $129 \mu \mathrm{m}$ with CAF-S4 (Fig. 6b). We next looked at the average distribution of cancer cell frequency along $z$-axis, every $10 \mu \mathrm{m}$ from the membrane up to $200 \mu \mathrm{m}$. Major part of cancer cells was below $30 \mu \mathrm{m}$, distance from which a major drop was observed (Fig. 6c). Interestingly, beyond $30 \mu \mathrm{m}$, CAF-S4 significantly increased the proportion of cancer cells compared to control (Supplementary Fig. 4e, left).
Similar results were obtained when increasing the invasion threshold, with an even greater impact of CAF-S4 on tumor cell invasion (Fig. 6d and Supplementary Fig. 4e, right). To go deeper in the characterization of $\mathrm{BC}$ cell motility in $3 \mathrm{D}$, we took advantage of the tumor-on-chip system ${ }^{42}$, a microfluidic device where cancer cells and CAF subsets were co-cultured in $3 \mathrm{D}$ and followed by time-lapse video microscopy. In this system, we reproduced the intrinsic CAF subset motility, CAF-S1 being more motile than CAF-S4 (Supplementary Fig. 4f). In addition, velocity of GFP-labeled BC cells increased in presence of CAF-S4, and at a lower extent CAF-S1 (Fig. 6e, f). In tumor-on-chip, CAF subsetmediated effect was more important on T47D (Fig. 6f) than MCF7 (Fig. 6e), probably because T47D cells are less motile at basal levels than MCF7 (around $0.1 \mu \mathrm{m} / \mathrm{min}$ for MCF7 and 0.05 $\mu \mathrm{m} / \mathrm{min}$ for T47D). Taken together, these results show that both CAF-S1 and CAF-S4 stimulate BC cell motility, via different effects: CAF-S1 initiate first steps of EMT and secrete factors that attract cancer cells, while CAF-S4 remodel the matrix and promote cancer cell invasion in $3 \mathrm{D}$.

CAF-S1 and CAF-S4 induce cancer cell invasion by TGF $\beta$ / CXCL12 and NOTCH. We next sought to define the molecular actors involved in CAF-S1 and CAF-S4 pro-invasive effects. As CAF-S1 attract cancer cell at distance and secrete high levels of CXCL12 (Supplementary Fig. 5a), we first analyzed the impact of CXCL12 silencing in CAF-S1 and CAF-S4 (Supplementary Fig. 5b) on $\mathrm{BC}$ cell proliferation and migration. Interestingly, CAF-S1 silenced for CXCL12 partly lost their ability to initiate EMT in cancer cells, while CXCL12-silencing had no impact in CAF-S4 (Fig. 7a). Indeed, BC cells were more clustered and Ecadherin protein levels in tumor cells increased after CXCL12 silencing in CAF-S1 but not in CAF-S4 (Fig. 7a and Supplementary Fig. 5c). We also observed that CXCL12-silencing in CAF-S1 decreased BC cell chemo-attraction without affecting their proliferation rate (Supplementary Fig. 5d, e). In agreement with these observations, even partial inhibition of CXCR4 in cancer cells (Supplementary Fig. 5f) affected their scattering and EMT initiation by CAF-S1 (Fig. 7b). This suggests that both CXCL12 secretion by CAF-S1 and CXCR4 expression in cancer cells are involved in CAF-S1-mediated EMT initiation in BC cells. As we observed an upregulation of the TGF $\beta$-signaling pathway in CAF-S1 (Supplementary Fig. 5g), we wondered whether this pathway, strong EMT inducer, could also be involved in CAF-S1mediated EMT initiation. As the expression of TGF $\beta$ receptors (TGFBR1, 2 and 3) was upregulated in CAF-S1 cells compared to CAF-S4 (Supplementary Fig. 5h), we compared the impact of TGFBR inhibitor on CAF-S1- and CAF-S4-induced EMT initiation on cancer cells (Fig. 7c and Supplementary Fig. 5i). TGFBR inhibition affected initiation of EMT in cancer cells in presence of 

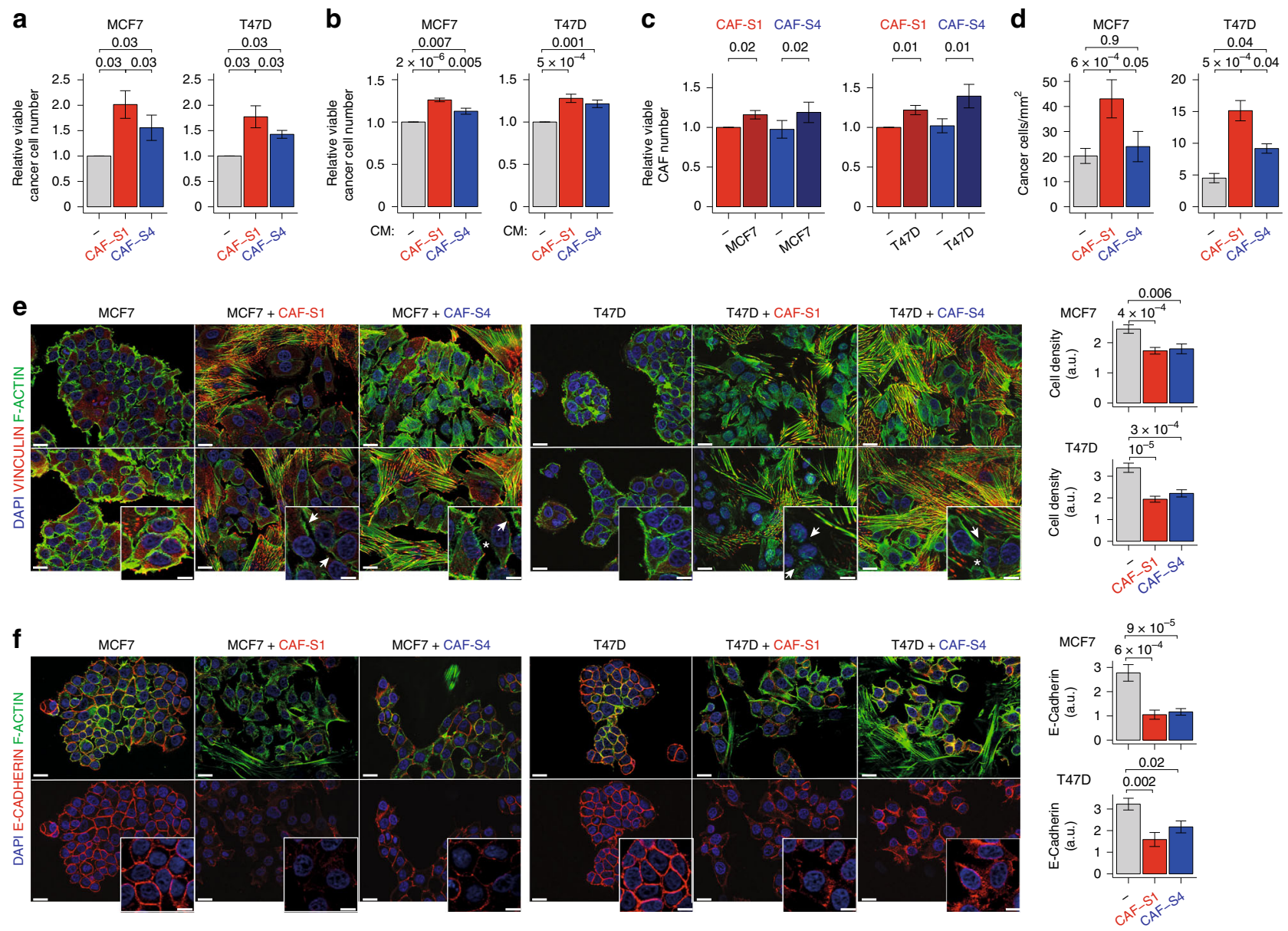

Fig. 5 CAF-S1 promote proliferation and initiate cancer cell epithelial-to-mesenchymal transition. a Total number of viable $B C$ cells (Dapi ${ }^{-}$cells by FACS) in co-culture with CAF-S1 (red) or CAF-S4 (blue) relative to control ( - gray, without CAF) ( $n=6$ per BC cell type). b Total number of viable BC cells (Resazurin staining) with CAF-S1- or CAF-S4-conditioned medium (CM) relative to control ( - , without CM) ( $n=9$ per BC cell type). c Total number of viable CAF-S1 (red) and CAF-S4 (blue) (Dapi- cells by FACS) in co-culture with BC cells relative to control (-, without BC cells) ( $n \geq 6$ per BC cell type). d CAF-S1 and CAF-S4 capacities to attract BC cells ( $n \geq 8$ per BC cells). e Left, Images of co-staining of Vinculin (red), F-actin (green) and DAPI (blue) in MCF7 (left) or T47D (right) cultured alone, or in presence of CAF-S1 or CAF-S4. Scale bars, $20 \mu \mathrm{m}$; inset, $10 \mu \mathrm{m}$. Arrows show reduced BC cell cohesion in presence of CAF-S1/-S4; asterisks show F-actin interconnections between BC cells in presence of CAF-S4. Vinculin and F-actin individual staining in Supplementary Fig. 4c. Right, Number of BC cells per tumor area (at least 13 images per condition, $n=3$ per BC cell type). $\mathbf{f}$ Left, Images of Ecadherin (red), F-actin (green) and DAPI (blue) co-staining (top) or of E-cadherin (red) and DAPI (blue) staining (bottom) in BC cells alone or in presence of CAF-S1/-S4. Scale bars, $20 \mu \mathrm{m}$; inset, $10 \mu \mathrm{m}$. Right, Quantification of E-cadherin staining per BC cell area (at least eight images per condition) ( $n=2$ per $\mathrm{BC}$ cell type). In all panels, barplots are mean \pm SEM and $n$ number of independent experiments; $a$. u., arbitrary units. a, $\mathbf{d}$ : $p$ Values from Wilcoxon signed rank test. b, c: $p$ Values from paired $t$-test. e right, f right: $p$ Values from Student's $t$-test (MCF7) and Mann-Whitney test (T47D). At least two CAF-S1 and CAF-S4 pairs tested, except in e, $\mathbf{f}$ for T47D, where one CAF-S1/-S4 pair is used. Source data provided in Source Data file, with R scripts used.

CAF-S1 cells, while it had no impact on cancer cells alone and a weak effect with CAF-S4 (Fig. 7c). Taken together, these results show the role of TGF $\beta$ and CXCL12 signaling pathways in CAFS1-dependent pro-tumorigenic phenotype.

Concerning CAF-S4, NOTCH pathway and three out of the four NOTCH receptors (NOTCH 1-3) were significantly upregulated in CAF-S4 (Supplementary Fig. 6a, b). We observed that DAPT, a $\gamma$-secretase inhibitor and pan-NOTCH inhibitor, severely reduced CAF-S4 contractile capacity assessed in collagen gel assays, while DAPT had no impact on CAF-S1 fibroblasts (Fig. 7d). Due to this strong effect of NOTCH inhibition on CAFS4 contractility, we next analyzed in-depth the effect of $\mathrm{NOTCH}$ on CAF-S4. We verified that DAPT had no effect on CAF-S4 viability (Supplementary Fig. 6c). As expected, Blebbistatin and Y27632, potent myosin and ROCK inhibitors used as positive controls, also abolished CAF-S4 contraction (Supplementary Fig. 6d). Using TFM, we confirmed that DAPT treatment also diminished the Strain Energy Density globally exerted by CAF-S4 cells (Fig. 7e), although it did not significantly affect the magnitude of the stress which averages the local traction forces (Fig. S6e). Moreover, the inverted Transwell invasion assay confirmed the role of NOTCH in CAF-S4-mediated BC cell invasion. Indeed, both the maximal distance reached and the percentage of invading tumor cells were reduced by NOTCH inhibition in CAF-S4-embedded matrix (Fig. 7f, h), while it had no or much lower impact without CAF-S4 (Supplementary Fig. 6f, g). Finally, using tumor-on-chip devices, we further showed that, although cancer cell motility was not affected by DAPT treatment in absence of CAF-S4, CAF-S4 were completely unable to induce BC cell invasion in presence of DAPT (Fig. 7i, j). Altogether, these data demonstrate that $\mathrm{NOTCH}$ signaling in CAF-S4 is essential to remodel the matrix and promote BC cell invasion in 3D. Finally, by using a cytokine array, we verified that the release of other chemokines and interleukins, such as IL6, IL8, CCL2, CCL5, known to be involved in crosstalk between CAF and cancer cells, was not statistically different between CAF-S1 and 
a
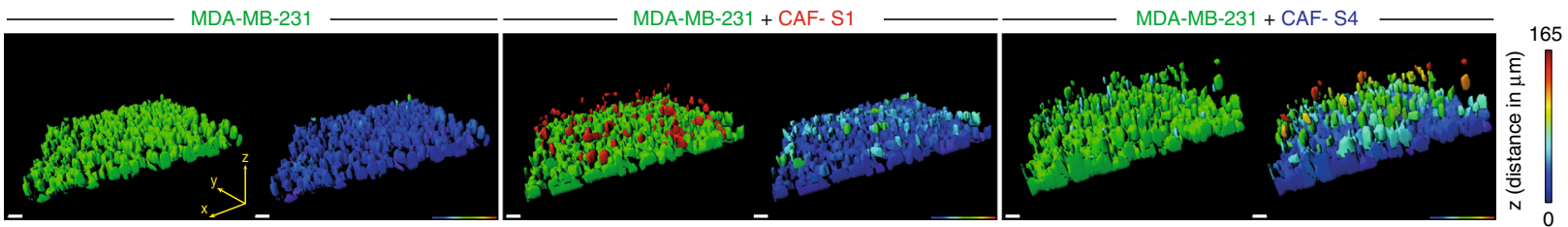

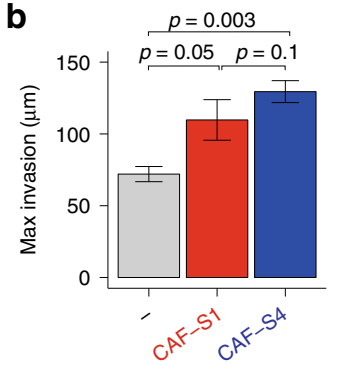

e

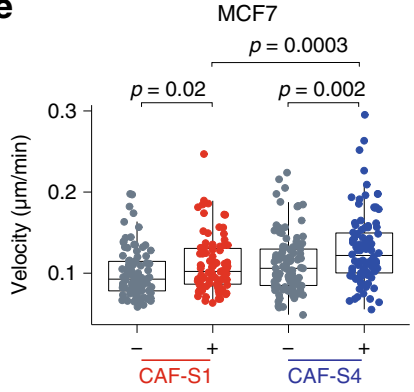

C

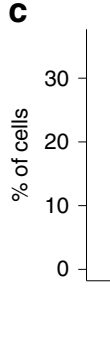

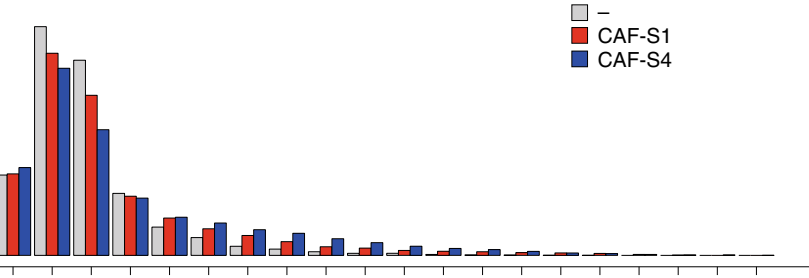

$102030 \quad 4050 \quad 6070 \quad 8090100110120130140150160170180190200$ $\mathrm{z}(\mu \mathrm{m})$
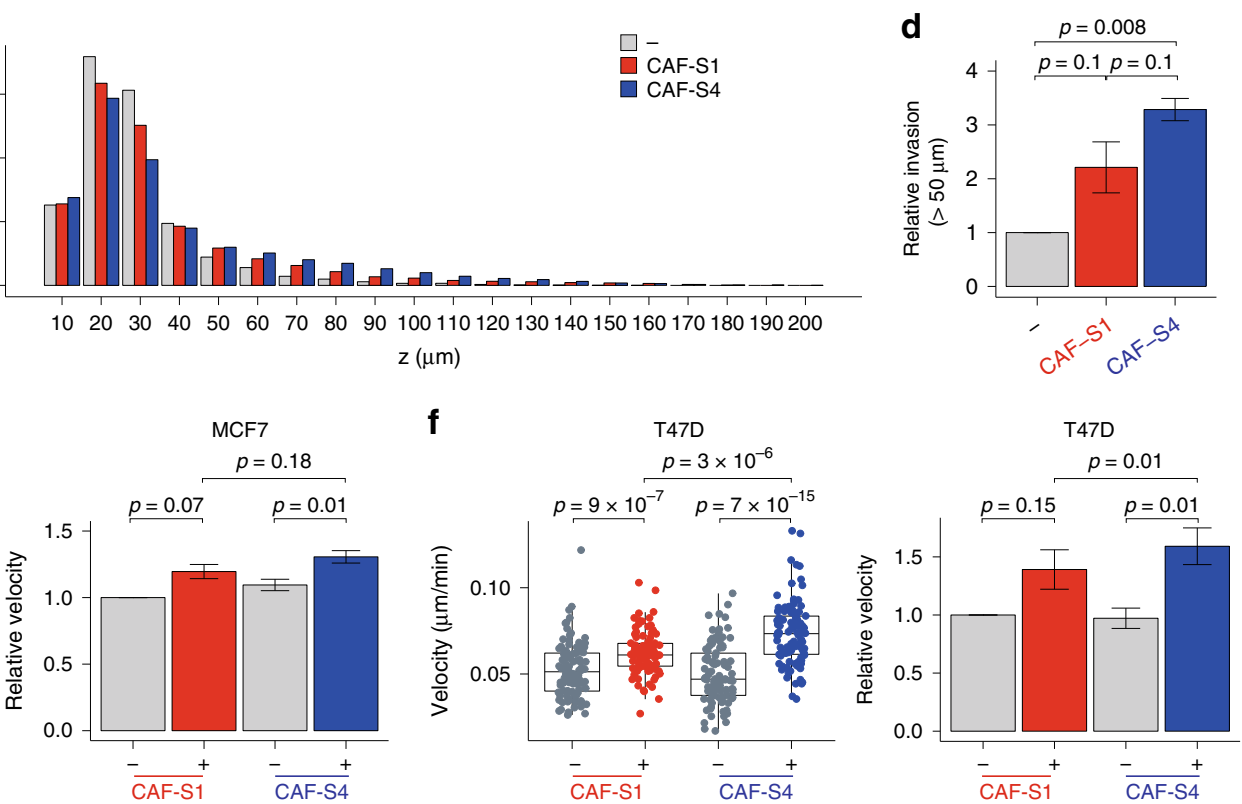

Fig. 6 CAF-S4 promote tumor cell invasion in 3D. a Representative 3D views of MDA-MB-231 invasion assessed by inverted Transwell assays, in CAFfree (left), CAF-S1- (middle) or CAF-S4- (right) embedded collagen matrix. In left images in each condition, colors show the different cell types: BC cells in green; CAF-S1 in red; CAF-S4 in blue. In right images in each condition, colors indicate the distance browsed by BC cells on the vertical ( $z$ ) axis in $3 \mathrm{D}$ (maximal distance $165 \mu \mathrm{m}$ in red). Scale bar, $50 \mu \mathrm{m}$. b Maximal vertical distance browsed by BC cells in CAF-free, CAF-S1- or CAF-S4-embedded collagen ( $n=3, \sim 500$ BC cells per analyzed $z$-stack). c Mean frequency (\%) of BC cells along vertical axis $(z, \mu \mathrm{m})$ in CAF-free, CAF-S1- or CAF-S4-embedded collagen. $\mathbf{d}$ Proportion of BC cells that invaded above $50 \mu \mathrm{m}$ in CAF-S1- or CAF-S4-embedded collagen, relative to CAF-free condition ( $n=3, \sim 500 \mathrm{BC}$ cells per analyzed z-stack). e Left, Representative tumor-on-chip experiment showing velocity of MCF7 in CAF-free, CAF-S1- or CAF-S4-embedded collagen. Each dot represents one cell ( $n \geq 90$ cells per condition). Right, Median velocity of MCF7 in CAF-S1- or CAF-S4-embedded collagen matrix relative to CAFfree condition $(n=3)$. f Same as in e for T47D. In all panels, barplots are mean \pm SEM. Boxplots are median $\pm 25 \%-75 \%$ quantiles, whisker values range $1.5 \times$ IQR above 75th or below 25th percentiles; $n$ indicates number of independent experiments. $\mathbf{b}, \mathbf{d}$, e right: $p$ Values from paired $t$-test. e left: $p$ Values from Mann-Whitney test. At least two CAF-S1 and CAF-S4 pairs have been tested. Source data provided in Source Data file, with R scripts used.

CAF-S4 (Supplementary Fig. 6i, j). In conclusion, CAF-S1 fibroblasts promote $\mathrm{BC}$ cell migration and EMT initiation in cancer cells in a CXCL12- and TGF $\beta$-dependent manner. In addition, CAF-S4 fibroblasts stimulate cancer cell invasion and motility in $3 \mathrm{D}$ by increasing contractility through the NOTCH signaling pathway.

CAF-S4-enriched LNs are associated with poor patient survival. We finally investigate the clinical relevance of our findings. We analyzed the impact of global stroma content and CAF subset enrichment in LN on disease-free survival, i.e. survival without local, regional or distant relapse (Fig. 8a and Table 1). As CAF-S1 are able to initiate EMT, we quantified the percentage of stromal and epithelial compartments in LNs. High content in stroma in LNs was associated with a shorter disease-free survival, with a similar tendency for overall survival ( $p=0.06$ by Log Rank test and $p=0.07$ by Cox regression model) (Fig. $8 \mathrm{a}, \mathrm{b}$ and Table 1 ). Using multivariate Cox regression analysis with additive model, we observed that the stromal content was independent of $\mathrm{BC}$ subtypes and LN status at diagnosis, two well-established independent prognostic parameters (Table 1). As invaded LNs are mainly composed of CAF-S1 or CAF-S4 (Fig. 2), these results suggested that both CAF subsets, when present in high quantity in invaded LNs at diagnosis, might be deleterious for patient survival. We found that CAF subsets on their own exhibited a faint but significant prognostic value, in particular when patients were stratified according to their stromal content in LNs (Fig. 8c and Supplementary Table 6). Indeed, among patients who displayed high stromal content in LNs at diagnosis, those enriched in CAF-S4 showed the poorest overall survival, while no difference according to CAF subset was observed in the context of low stromal content (Fig. 8c). Of note, the prognostic value of CAF subset content in LNs is interesting-although faint-as it is not observed in $\mathrm{PT}^{32,33}$. Considering that CAF-S1 and CAF-S4 enhance BC cell invasive capacities and that most BC patients decease from metastases, we next looked at distant metastases developed after diagnosis. In line with our results, patients who developed such metastases were mainly patients with CAFabundant LNs at diagnosis, with a similar repartition in $\mathrm{BC}$ subtype (Fig. 8d). The impact on distant metastatic spread was even stronger in CAF-S4- than in CAF-S1-enriched LN patients, effect detected in all BC subtypes (Fig. 8e). Overall, patients who showed LNs with high stromal content and specific enrichment in CAF-S4 were more prone to develop late distant metastases than any other patients, in particular in liver (Fig. 8f, g). Although metastatic sites vary according to $\mathrm{BC}$ subtypes with bone as the 
a
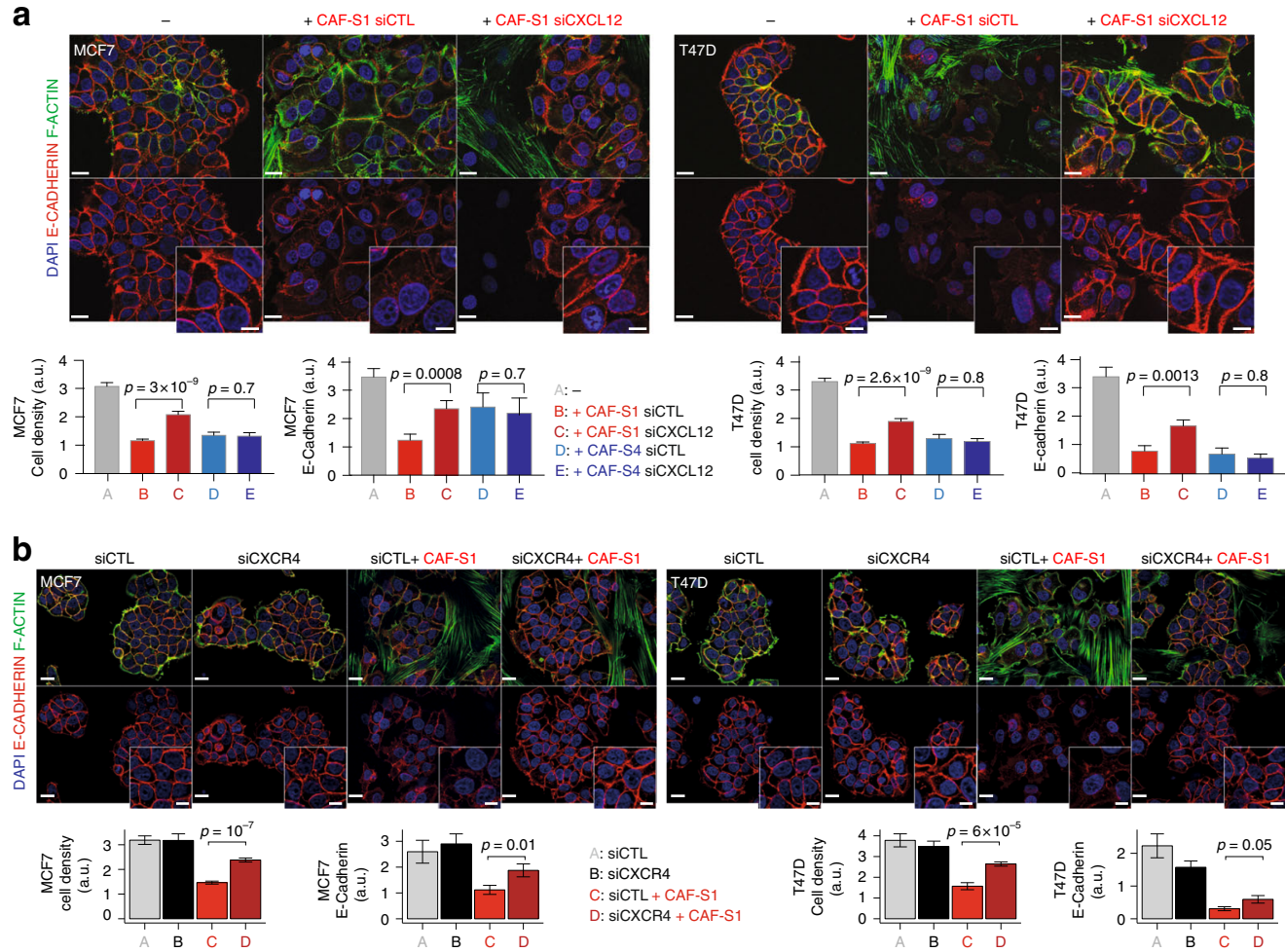

\section{C}
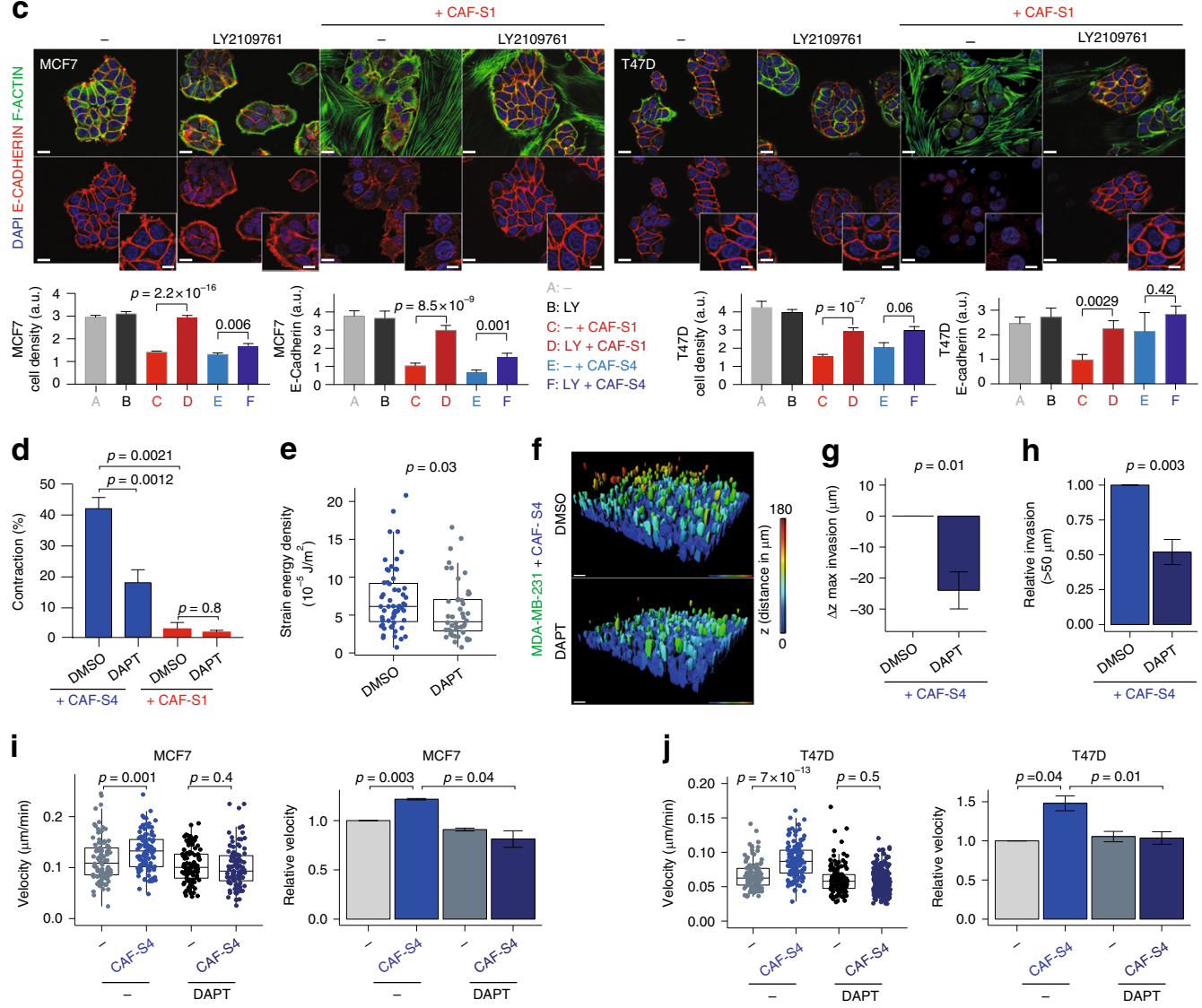

most common site except in TN BC associated with other niches, such as liver ${ }^{1,2}$, CAF-S4 impact was not associated to a specific $\mathrm{BC}$ subtype. Indeed, among all patients displaying late liver metastasis, only one patient (6\%) presented HER2 overexpression at diagnosis. Moreover, CAF-S4-enriched metastatic patients were numerous both within Lum and TN BC subtypes (Fig. 8g). We even detected a higher proportion of patients categorized as CAF-S4-enriched with high stromal content in LNs in the Lum BC subtype compared to TN BC patients (60\% Lum versus $33 \%$ $\mathrm{TN})$. Thus, patients with high-CAF-S4 content in LNs, who 
Fig. 7 CAF-S1 and CAF-S4 promote cancer cell invasion by TGF $\beta$, CXCL12 and NOTCH. a Up, Representative images of E-cadherin (red), F-actin (green) and DAPI (blue) in BC cells alone (-) or with CAF-S1 transfected with non-targeting (siCTL) or CXCL12-targeting (siCXCL12) siRNA. CAF-S4 images in Supplementary Fig. 5c. Scale bars, $20 \mu \mathrm{m}$; inset $10 \mu \mathrm{m}$. Down, BC cell density and E-cadherin staining alone (gray) or with CAF (CAF-S1 red; CAF-S4 blue) ( $\geq 12$ images/condition; $n=8$ ). b Same as a with siCTL- or siCXCR4-transfected BC cells, with/without CAF-S1 (red/gray) ( $\geq 8$ images/condition; $n=4)$. c Same as a with/without CAF-S1 with/without TGF $\beta$-R inhibitor (LY2109761). CAF-S4 images in Supplementary Fig. 5e. Quantifications without (gray) or with CAF (CAF-S1 red; CAF-S4 blue) ( $\geq 7$ images/condition; MCF7: $n=7 ;$ T47D: $n=3$ ). d Percentage (\%) of collagen contraction by CAF-S4 (blue) or CAF-S1 (red) without (DMSO) or with DAPT $(n \geq 3)$. e Strain energy density of CAF-S4 without (DMSO, blue) or with DAPT (gray). Each dot is one cell, $n \geq 46$ cells/condition. $\mathbf{f}$ Representative 3D views of MDA-MB-231 (green) invasion by inverted Transwell assays in CAF-S4 (blue)-embedded collagen matrix without (DMSO) or with DAPT. Colors indicate distance browsed by BC cells on $z$ axis ( $d_{\max } 180 \mu \mathrm{m}$, red). Scale bar, $50 \mu \mathrm{m}$. $\mathbf{g}$ Maximal distance of BC cells in CAF-S4-embedded collagen with DAPT relative to control (DMSO) ( $n=6, \sim 700$ BC cells/z-stack). $\mathbf{h}$ Same as in $\mathbf{g}$ for proportion of BC cells that invaded above $50 \mu \mathrm{m}$. i Left, Velocity of MCF7 in tumor-on-chip in CAF-free (-, gray) or CAF-S4 (blue)-embedded collagen treated or not with DAPT. Each dot is one cell, $n \geq 95 /$ condition. Right, Same as left for median velocity of MCF7 $(n=3)$. j Same as $\mathbf{i}$ for T47D. In all panels, boxplots are median \pm 25\%-75\% quantiles, whisker values range $1.5 \times$ IQR above 75th or below 25th percentiles. Barplots mean \pm SEM. a-e $p$ Values from Mann-Whitney test. g-i $p$ Values from paired $t$-test. At least four CAF-S1 and CAF-S4 tested. Source data provided in Source Data file, with R scripts used.

tended to metastasize into liver, were not enriched in TN BC subtype in the cohort analyzed. The impact of CAF subset on metastatic spread was thus not related to a bias linked to BC subtype. On the whole, these data highlight the role of CAF-S1 and particularly CAF-S4 subsets in human BC relapse and metastases (see Model Fig. 8h).

\section{Discussion}

Although the role of CAF in metastatic spread is well established, when analyzed as a global population $9,12,14,18,20,43$, we address here the link between CAF subsets and BC cell spread. Axillary metastatic LN exhibit four CAF subsets (CAF-S1 to S4) but are highly enriched in aSMA+ CAF-S1 and CAF-S4 subsets. The abundance of $\alpha \mathrm{SMA}+\mathrm{CAF}$ at PT has been associated with poor prognosis in $\mathrm{BC}^{17,44-48}$. In agreement with our findings, a recent study reported that the global stromal content in metastatic LN provides a prognostic stratification of $\mathrm{BC}$ patients ${ }^{49}$. We go a step further by differentiating CAF-S1 and CAF-S4 and by showing that the CAF-S1/CAF-S4 status in LNs exhibits a prognostic value, while this is not the case when considering CAF subset enrichment in $\mathrm{PT}^{9,12,14,18,20,43}$. This underlines the relevance of our study in BC pathology and indicates that assessing the content in CAF-S1 and CAF-S4 subsets in LNs might be helpful for clinical diagnosis.

Both CAF-S1 and CAF-S4 subsets exhibit pro-invasive properties yet with distinct modes of action. CAF-S1 enhance tumor cell migration and EMT initiation, while CAF-S4 favor cancer cell invasion and motility in 3D. Although the impact of CAF in metastatic spread has been previously demonstrated ${ }^{50-53}$, we show here the complementary role of two distinct CAF subpopulations in metastases. We identify a crosstalk between CAF$\mathrm{S} 1$ and cancer cells involving both CXCL12 and TGF $\beta$, consistent with data showing that CAFs secrete CXCL12 and promote cancer cell migration $16,17,54-58$. In addition, we highlight the role of TGF $\beta$, a well-known EMT inducer ${ }^{4,59,60}$ and an important player in fibroblast activation ${ }^{50,61-63}$. Both CXCL12 and TGF $\beta$ expression are found in $\mathrm{BC}$ metastatic sites, in particular lung and bones, supporting they are particularly relevant in BC. In contrast to CAF-S1, CAF-S4 are highly contractile and exert a pro-invasive effect on cancer cells. Although mechanisms linking CAF contractility to cancer cell invasion have been described $22,25,64,65$, we unveil here the NOTCH pathway as a key player. NOTCH pathway has been studied in cancer with a strong focus on cancer cells $^{66-68}$. Our data show that NOTCH exerts a key role in CAFS4 contractility and its pro-invasive action in BC. Thus, we report specific CAF-S1 and CAF-S4-mediated processes and mechanisms that promote metastatic spread in $\mathrm{BC}$.

The four CAF subsets we identified in LN mirror CAF subpopulations in $\mathrm{PTs}^{32,33}$. This is consistent with studies showing that LN stroma mimics PT microenvironment ${ }^{28,30}$. But, our results provide new insights. Indeed, if the four CAF subsets are detected in both PT and LN, their abundance strikingly differs. Compared to PT, LNs are almost exclusively enriched in activated CAF-S1 and CAF-S4. The transcriptomic signatures of CAF subsets from LN validated molecular similarities between CAF-S1 (or CAF-S4) from PT and LN. If few studies addressed CAF transcriptomic profiles in both PTs and LNs from BC patients $^{37,69}$, a short gene list identified in ref. ${ }^{37}$ allows to discriminate LN from PT bulk samples. Interestingly, most of these genes are highly detected in CAF-S4, consistent with their abundance in metastatic LNs. Although CAF origin remains an open question, CAF-S1 and CAF-S4 transcriptomic profiles generated from PT and LN indicate that subset identity is similar between the two tissues. A recent study identified nine stromal populations by single-cell RNAseq in mouse $\mathrm{LN}$, including the established FRCs, FDC and perivascular cells ${ }^{70}$. Although these cells were identified in non-cancerous mouse lymphoid tissues, CAF-S1 are close to CD34+ stromal cells and CAF-S4 to perivascular cells. Our data on CAF-S4 are consistent with previous studies showing the existence of pericyte-like CAF and their potential pericytes-derived origin ${ }^{32,38-41}$. Thus, CAF subsets in invaded LNs could derive from different resident mesenchymal cells. In conclusion, by combining the study of BC patient samples and performing functional assays on human CAF subset cultures, we demonstrate that CAF subsets exhibit distinct functions in metastatic spread. Considering the deleterious effect of metastases on BC patients' survival, our data might strengthen the interest in assessing CAF subsets content in LN for clinical diagnosis and in using anti-TGF $\beta$ and/or anti-NOTCH therapies in $\mathrm{BC}$.

\section{Methods}

Cohorts of BC patients. The projects developed here are based on surgical residues, available after histopathological analyses, and not required for diagnosis. There is no interference with clinical practice. Analysis of PT and metastatic LN samples was performed according to the relevant national law on the protection of people taking part in biomedical research. All patients included in our study were informed by their referring oncologist that their biological samples could be used for research purposes and they gave their verbal informed consent. In case of patient refusal, that could be either orally expressed or written, residual tumor samples were not included in our study. Human experimental procedures were approved by the Institutional Review Board and Ethics committee (CRI) of the Institut Curie Hospital group (approval 12 February 2014) and CNIL (Commission Nationale de l'informatique et des Libertés) (No. approval: 1674356 delivered 30 March 2013). HER2-amplified carcinomas have been defined according to ERBB2 immunostaining using ASCO's guideline. Luminal (Lum) tumors were defined by positive immunostaining for ER (estrogen receptor) and/or PR (progesterone receptor). The cut-off used to define hormone receptor positivity was $10 \%$ of stained cells. Ki67 (proliferation) score further distinguishes Lum A from Lum B tumors (below 15\%: Lum A, above: Lum B). TN immunophenotype was defined as
} 
a

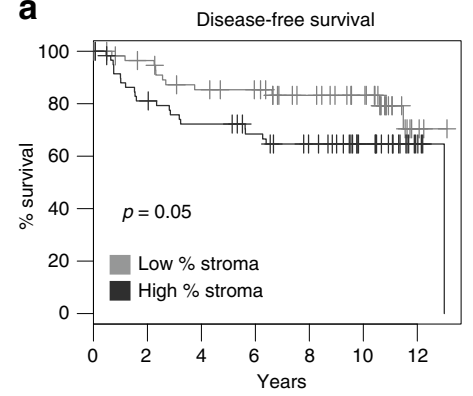

d
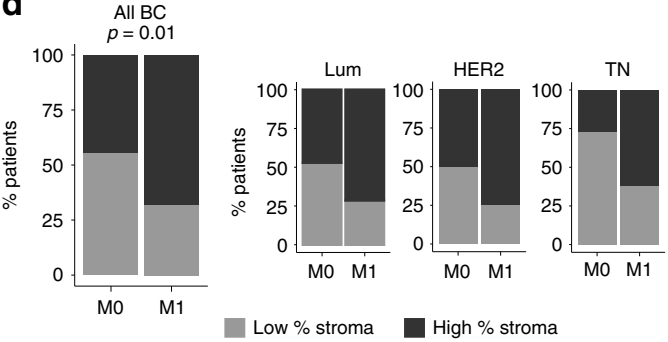

b

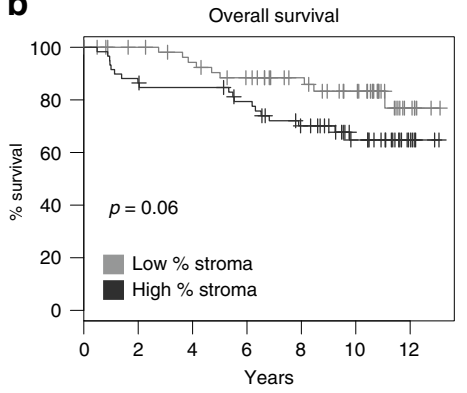

C

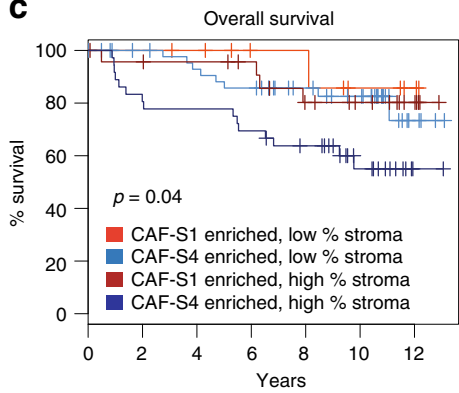

e $\quad$ All BC
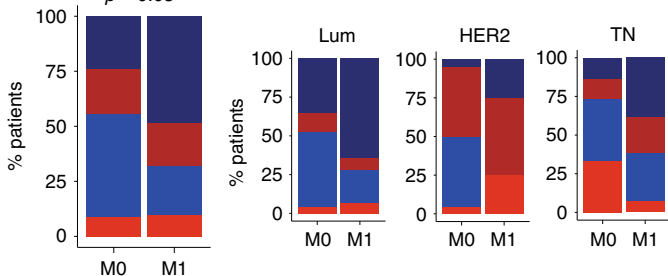

CAF-S1 enriched, low \% stroma CAF-S1 enriched, high \% stroma

CAF-S4 enriched, low \% stroma CAF-S4 enriched, high \% stroma

f

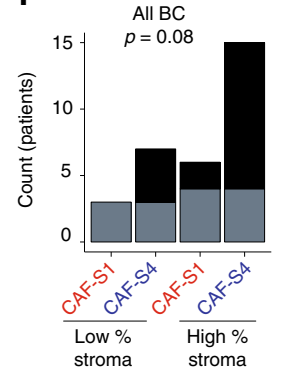

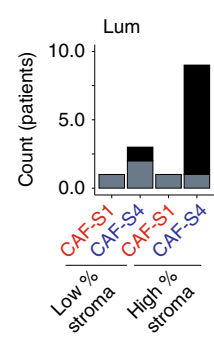

HER2

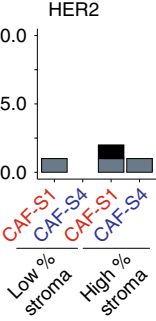

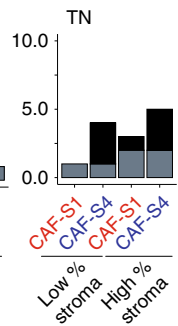

With liver metastases g

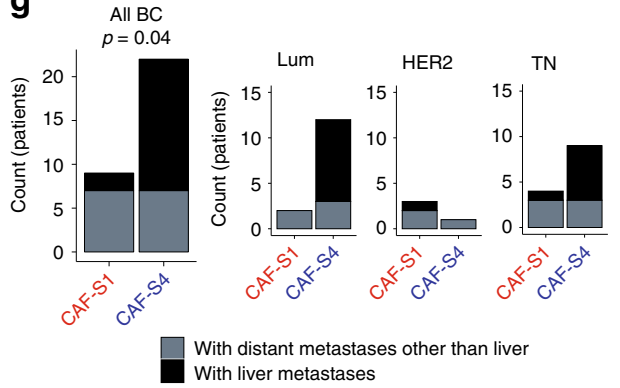

h

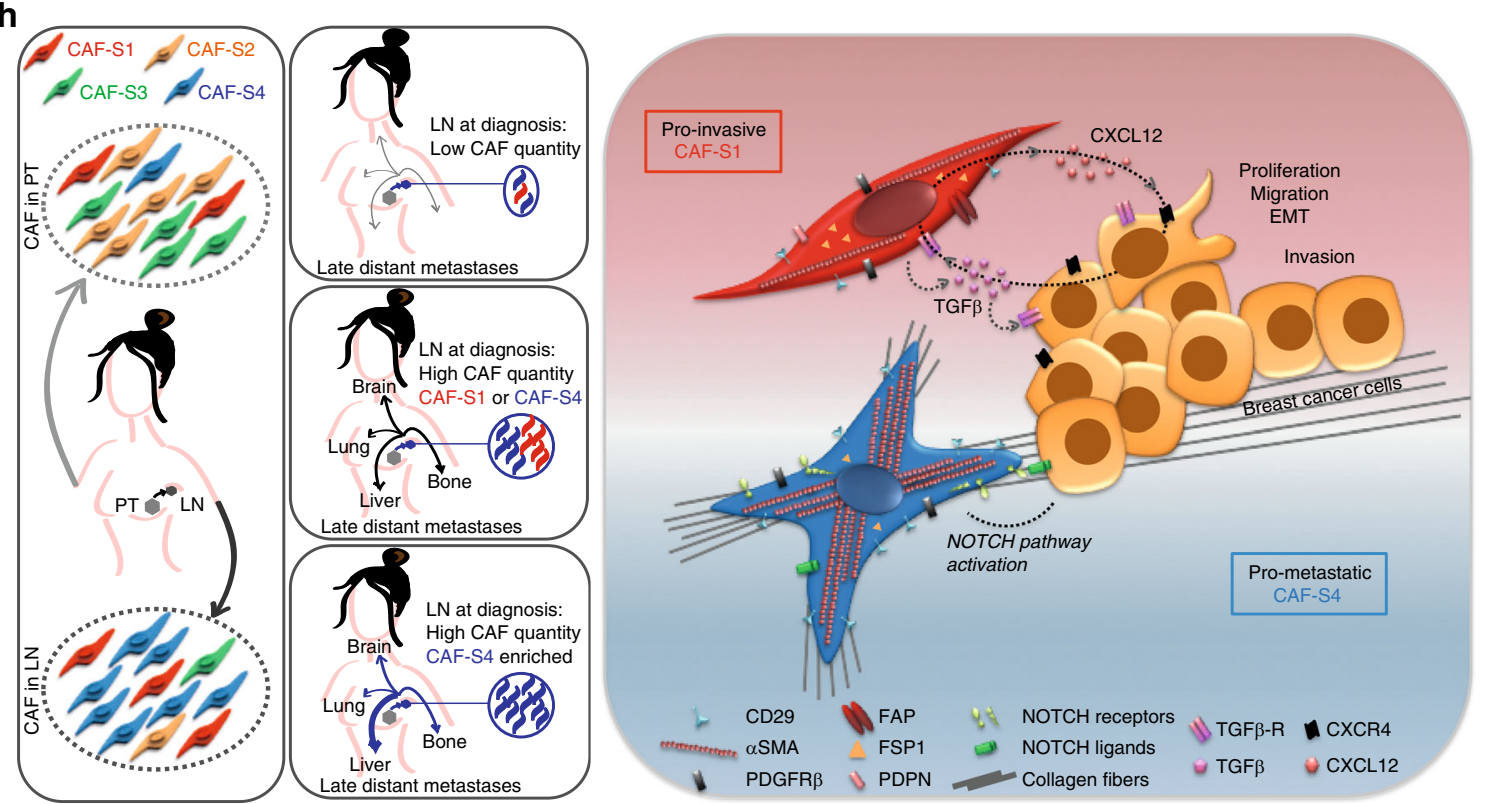

follows: $\mathrm{ER}^{-} \mathrm{PR}^{-} \mathrm{ERBB}^{-}$with the expression of at least one of the following markers: $\mathrm{KRT}^{-} / 6^{+}$or EGF- ${ }^{+}$.

BC PTs and metastatic LNs from prospective cohorts were collected as part of routine standard of care and included in this study after evaluation by a pathologist. CAF subsets were analyzed from fresh PT and LN samples by FACS (16 PTs and 20 LN), RNA sequencing (5 PTs and their corresponding LN), and cultured in vitro (14 $\mathrm{PT}$ and $8 \mathrm{LN})$. Prospective cohorts mainly include Lum subtypes.
PT (up to 75) and LN samples (up to 124) from retrospective cohorts of BC patients suffering from invasive BC cancers with at least one metastatic LN at diagnosis, have been collected as part of routine standard of care and analyzed by IHC. Retrospective cohorts include Lum, HER2 and TN subtypes. IHC were performed on residual surgery samples prior to any treatment (i.e. prior to radiation, hormonal or chemo-therapy). Overall survival and disease-free survival have been defined as followed: overall survival is defined as the period of time going 
Fig. 8 CAF subset content in LNs is a prognostic marker. a Kaplan-Meier curves showing patient disease-free survival according to percentage (\%) of stroma relative to epithelium in $L N$ sections $(n=119)$. Patient subgroups defined by median. $\mathbf{b}$ Same as in a for overall survival. c Overall survival according to both LN stromal quantity (as in a) and CAF-subset enrichment, as defined in Fig. $2(n=119)$. a-c $p$ Values from log rank test. d Left, Distribution of patients with low- or high-LN stromal quantity (as in a) without (MO, $n=88$ ) or with $(M 1, n=31)$ metastases. Right, Same as in left per BC subtypes (65 Lum; 26 HER2; 28 TN). e Same as in $\mathbf{d}$ according to LN stromal quantity and CAF-subset enrichment (same groups as in $\mathbf{c}$ ). $\mathbf{f}, \mathbf{g}$ Left, Number of patients with metastases in any distant site except liver (gray) or with at least one metastasis in liver (black), according to CAF content in LNs at diagnosis ( $n=31$ ) either considering both CAF quantity and CAF-subset enrichments (same groups as in $\mathbf{c}$ ) (f) or considering CAF-S1 and CAF-S4 enrichments (g).

d-g $p$ Values from Fisher's exact test. h Model: Left: four CAF subsets (CAF-S1 to -S4) are detected in metastatic axillary LN, as in PT. Two myofibroblast subsets (CAF-S1, red and CAF-S4, blue) are highly abundant in invaded LNs and correlated with tumor cell invasion. Middle: While patients with low-CAF content in LNs at diagnosis have less risk to develop late distant metastasis, those presenting high CAF-S1/S-4 quantity are prone to develop distant metastases, in particular in liver if LNs are CAF-S4-enriched. Right: Both CAF-S1 and CAF-S4 display pro-invasive properties through distinct mechanisms. CAF-S1 promote cancer cell proliferation, attraction and EMT initiation through CXCL12-CXCR4 and TGF $\beta$ axes. Highly contractile and matrix remodeler CAF-S4 induce cancer cell invasion in 3D via NOTCH signaling pathway. Thus, our work reveals the clinical interest of defining CAF subsets content in LNs at diagnosis. Source data provided in Source Data file, with $\mathrm{R}$ scripts used.

from diagnosis to death of the patient; disease-free survival is the period of survival without local, regional or distant relapse, meaning the period of time going from diagnosis to the first event occurring among local, regional or distant relapse.

Clinical features of prospective and retrospective cohorts are listed in Tables S1 and S2.

Flow cytometry on BC PT and LN samples. Fresh human BC PT and LN samples were collected directly from the operating room, after surgical specimen's macroscopic examination and selection of areas of interest by a pathologist. Samples were cut into small pieces (around $1 \mathrm{~mm}^{3}$ ) and digested in $\mathrm{CO}_{2}$-independent medium (Gibco \#18045-054) supplemented with $150 \mu \mathrm{g} / \mathrm{ml}$ liberase (Roche $\# 05401020001$ ) and DNase I (Roche \#11284932001) during $40 \mathrm{~min}$ at $37^{\circ} \mathrm{C}$ with shaking $(180 \mathrm{rpm})$. Cells were then filtrated through a $40-\mu \mathrm{m}$ cell strainer (Fisher Scientific \#223635447) and resuspended in PBS+ solution (PBS, Gibco \#14190; EDTA 2 mM, Gibco \#15575; Human Serum 1\%, BioWest \#S4190-100) at a final concentration between $5 \times 10^{5}$ and $10^{6}$ cells in $50 \mu \mathrm{l}$.

Cells in suspension were then stained with an antibody mix containing antiEpCAM $^{-}$BV605 (1:50; BioLegend, \# 324224), anti-CD31-PECy7 (1:100, BioLegend, \#303118), anti-CD45-APC-Cy7 (1:20, BD Biosciences, \#BD-557833), anti-CD235a-PerCP/Cy5.5 (1:50, Biolegend, \#349109), anti-CD29-Alexa Fluor 700 (1:100, BioLegend, \#303020), anti-FAP-APC (primary antibody, 1:100, R\&D Systems, \#MAB3715), anti-PDGFR $\beta$-PE (1:40, BioLegend, \#323606) and antiPDPN-Alexa Fluor 488 (1:50, BioLegend, \#337006) for surface staining and antiSMA-Alexa Fluor 594 (1:25, R\&D Systems, \#IC1420T-025) for intracellular staining. All antibodies except FAP were purchased already conjugated with fluorescent dyes. Anti-FAP antibody was conjugated with fluorescent dye Zenon APC Mouse IgG1 labeling kit (1:100, Thermo Fisher Scientific, \#Z25051). Isotype control antibodies for each CAF marker used were: iso-anti-CD29 (1:100, BioLegend, \#400144), iso-anti-FAP (primary antibody, 1:200, R\&D Systems, \#MAB002), iso-anti-PDGFR $\beta$ (1:20, BioLegend, \#400114), iso-anti-PDPN (1:125, BioLegend, \#400525) and iso-anti-SMA (1:25, R\&D Systems, \#IC003T). For intracellular staining, cells were incubated with violet LIVE/DEAD dye (1:1000, Thermo Fisher Scientific, \#L34955) for $20 \mathrm{~min}$ at room temperature (RT) in PBS (Gibco, \#14190) to exclude dead cells, and then fixed in $4 \%$ paraformaldehyde (PFA, Electron Microscopy Sciences, \#15710) overnight at $4{ }^{\circ} \mathrm{C}$. After a rapid washing step in PBS + solution, cells were incubated for $45 \mathrm{~min}$ at RT with the antibody mix in PBS + supplemented with $0.1 \%$ saponin (Sigma-Aldrich, \#S7900). For surface staining, cell suspensions were stained immediately after dissociation of BC PT and LN samples during 15 min at RT with the antibody mix in PBS+ solution. $2.5 \mu \mathrm{g} / \mathrm{ml}$ DAPI (Thermo Fisher scientific, \#D1306) was added just before flow cytometry analysis. For primary CAF-S1 and CAF-S4 culture characterization, cells at $80 \%$ confluency were collected after trypsin treatment, resuspended in PBS + solution at a final concentration of $5 \times 10^{5}$ cells in $50 \mu \mathrm{l}$ and intracellularly stained with the above-described protocol.

In both conditions (surface and intracellular staining), signals were acquired on the LSRFortessa ${ }^{\mathrm{TM}}$ analyzer (BD biosciences) for flow cytometry analysis. At least $5 \times 10^{5}$ events were recorded. Compensations were performed using single staining on anti-mouse IgG and negative control beads (BD biosciences, \#552843) for each antibody except anti-PDPN, on ArC reactive beads (Molecular probes \#A10346) for live/dead staining and on AbC Total Antibody Compensation Beads (Thermo Fisher Scientific, \#A10513) for anti-PDPN.

Data analysis was performed using FlowJo version X 10.0.7r2. Cells were first gated based on forward (FSC-A) and side (SSC-A) scatters (measuring cell size and granularity, respectively) to exclude debris. Dead cells were excluded based on their positive staining for Live/Dead (fixed conditions) or DAPI (surface staining). Single cells were next selected based on SSC-A versus SSC-W parameters. Gating included $\mathrm{EPCAM}^{-}, \mathrm{CD}^{-} 5^{-}, \mathrm{CD} 31^{-}, \mathrm{CD} 235 \mathrm{a}^{-}$cells, to remove epithelial $\left(\mathrm{EPCAM}^{+}\right)$, hematopoietic $\left(\mathrm{CD}^{2} 5^{+}\right)$, endothelial $\left(\mathrm{CD} 31^{+}\right)$and red blood cells $\left(\mathrm{CD} 235 \mathrm{a}^{+}\right)$. Cells from the negative fraction were next examined using the 5 CAF markers FAP, CD29, $\alpha$ SMA, PDGFR $\beta$ and PDPN.
FlowSom algorithm was performed using R packages FlowSOM (1.12.0) and flowCore (1.462), according to the methodology described in ref. ${ }^{35}$. SelfOrganizing Map was performed on all $\mathrm{EPCAM}^{-}, \mathrm{CD}^{-} 5^{-}, \mathrm{CD} 31^{-}, \mathrm{CD} 235 \mathrm{a}^{-}$cells from 16 PTs and from 20 LNs to generate unified trees based on the expression of the five CAF markers. CAF subset populations were manually annotated.

IHC analyses. BC PT and LN cases were selected by Pathologists (see Supplementary Table 2) and serial sections of paraffin-embedded tissues (PT: TissueMicro-Arrays (TMA) using two cores $(1 \mathrm{~mm}$ diameter, selected as representative from whole-tumor tissue sections by Pathologists) of tumor per case and cut in slices of $3 \mu \mathrm{m}$; LN: whole sections of 3- $\mu \mathrm{m}$ thickness) were stained on the Lab Vision Autostainer (Thermo Fisher Scientific). Dewaxing and antigen retrieval on slides prior to immunohistochemical staining were performed with EnVision FLEX Target Retrieval Solution (high- or low-pH, as required-see below-Dako, \#K800421 or \#K800521) on the Lab Vision PT Module (Thermo Fisher Scientific). Antigen detection was done using the EnVision FLEX/HRP (Dako, \#K8006) for CD29, $\alpha$ SMA and PDGFR $\beta$ or the streptavidin-peroxidase protocol (Vectastain ABC kit; Vector Labs \#PK-6101/6102/6104) plus detection with 3,3'-diaminobenzidine for 5 min (DAB, Dako, \#K3468) for FSP1, EPCAM (on LN) and FAP. The following antibodies and respective conditions were used: anti-FAP $(\mathrm{pH}=6$, $1 \mathrm{~h}$ at 1:200, Vitatex \#MABS1001), anti-CD29 $(\mathrm{pH}=6,1 \mathrm{~h}$ at 1:100, Abcam \#ab3167), anti-FSP1 ( $\mathrm{pH}=6,1 \mathrm{~h}$ at 1:250, Abcam \#ab27957), anti- $\alpha \mathrm{SMA}(\mathrm{pH}=6$, $30 \mathrm{~min}$ at $1: 200$, Dako \#M0851), anti- PDGFR $\beta$ ( $\mathrm{pH}=9,1 \mathrm{~h}$ at $1: 100$, Abcam \#ab32570), anti-EPCAM ( $\mathrm{pH}=6,1 \mathrm{~h}$ at 1:200, Dako \#M0804), anti-E-cadherin $(\mathrm{pH}=9,1 \mathrm{~h}$ at 1:100, Invitrogen \#180223). Counterstaining was performed with Mayer hematoxylin freshly prepared (Dako, \#S3309). Tissue sections were then submitted to serial gradients of xylen and mounted with coverslip in an automatic device (Sakura, Tissue-Tek DRS).

In each sample, CAF marker staining was evaluated as a histological score $(\mathrm{H}-$ score), defined by staining intensity (ranging from 0 to 4 ) multiplied by the percentage of stained fibroblasts. For LNs, only fibroblasts in metastatic zonesassessed by EPCAM staining-were evaluated. For each PT and LN sample, CAF enrichment was established applying an algorithm developed by the team, which takes as input CAF markers H-scores, as described in refs. ${ }^{32,33}$. In brief, the thresholds were first defined in a learning dataset on the distribution (1st quartile, median and 3rd quartile) of each marker using FACS data. Thresholds were then transposed to IHC data. For each section, the percentages of stroma and epithelial compartments were evaluated by combining three distinct methods: evaluation on morphological criteria by a referent pathologist; quantification with an automated approach using the QuantCenter image analysis platform (3DHistech) based on morphology assessment and EPCAM staining performed by an independent researcher; EPCAM and E-cadherin staining of epithelial cells. All quantifications gave very consistent results whatever the person (pathologist versus researcher), the method (manually versus automated) or the staining (EPCAM/E-cadherin), thereby arguing that evaluation of stromal and epithelial content was accurate. In each section, percentage of stroma was evaluated as the fibroblastic area multiplied by 100 divided by the total sample area.

Design of a decision tree for CAF subset prediction. CAF identity was determined by using an algorithm developed by the team (shown in Fig. 2d), which takes as input histological scores of CAF markers ${ }^{32,33}$. The thresholds were first defined, in a learning dataset, on the distribution (1st quartile, median and 3rd quartile) of each marker using FACS data. Thresholds were then transposed to IHC data.

CAF subsets and epithelial cells at cellular level in situ in invaded LNs. IHC staining from consecutive sections were scanned on Philips Ultra Fast Scanner; $5 \times$ images of CAF markers including FAP, CD29, FSP1 and SMA (markers used in the decision tree algorithm, see also Fig. 2d) as well as EPCAM staining from the same 


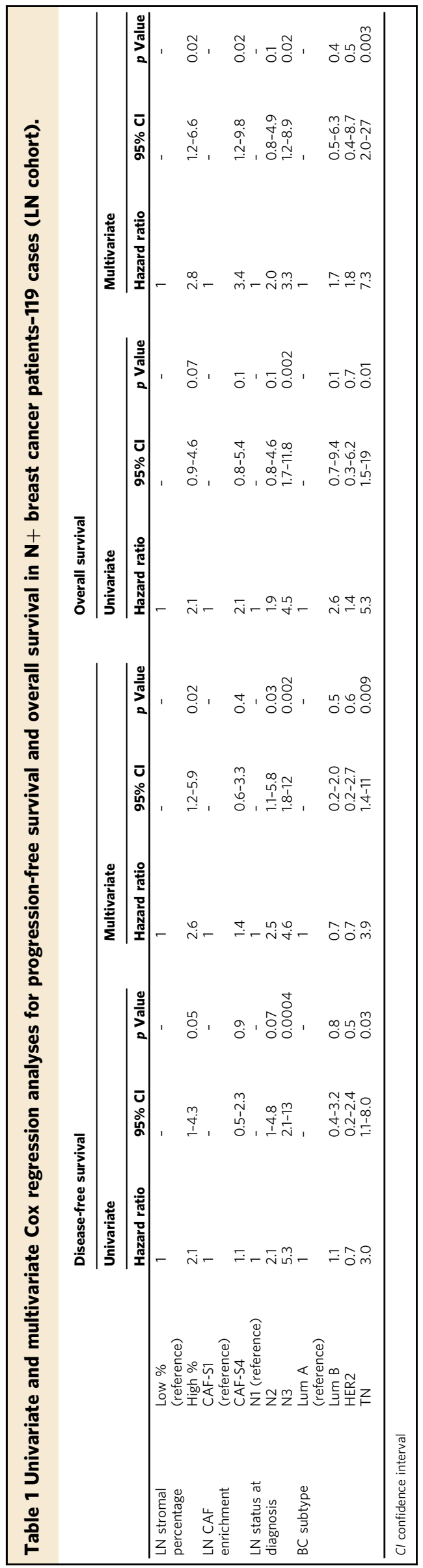

areas in representative invaded LNs were further analyzed. Images were aligned using elastic transformation from Fiji software plugin (bUnwarpJ). This plugin uses landmarks manually defined on hematoxylin \& eosin staining of the sections to compute the optimal correlation between images and alignment at cellular level. Images were divided into tiles of $225 \mu \mathrm{m}^{2}$ to mimic the approximate size of one fibroblast and each tile was annotated according to the position in the section. Aligned and annotated images of the CAF markers were then submitted to color deconvolution and the intensity of each DAB staining was measured by densitometry analysis using ImageJ software. Each tile was classified into a specific CAF subset using the algorithm developed by the team (see 'Design of a decision tree for CAF subset prediction' and Fig. 2d), which takes as input DAB intensities of CAF markers measured within each tile. Epithelial tumor cells were detected based on EPCAM staining to better visualize the stromal compartment and each tile was colored according to the classification into CAF-S1 to CAF-S4, with CAF-S1 red, CAF-S2 orange, CAF-S3 green and CAF-S4 blue.

Gene expression profiling by RNAseq. CAF-S1, CAF-S4 and $\mathrm{EPCAM}^{+}$cells (from PTs and LNs of five patients, $n=28$ samples in total, see Supplementary Table 1) were sorted after surface staining (see 'Flow Cytometry on BC PT and LN samples') on FACSARIA (BD biosciences), directly into RNase-free tubes (Thermo Fisher Scientific \#AM12450). At least 100 cells were collected per cell population. Total RNA was extracted with Single Cell RNA Purification kit (Norgen Biotek \#51800) following manufacturer's instructions. RNA integrity and quality were checked with Agilent RNA 6000 Pico Kit (Agilent, \#5067-1513) and apparatus. cDNA synthesis and amplification were synthetized using SMART-Seq v4 Ultra Low Input RNA Kit for Sequencing (Clontech \#634892). cDNA quality was checked on Agilent 2100 Bioanalyzer using Agilent High Sensitivity DNA kit (Agilent \#5067-4626) and quantified using the Qubit dsDNA HS Assay kit (Life Technologies, \#Q32854). cDNA libraries were prepared using Nextera XT preparation kit (Illumina \#FC-131-10). Samples were sequenced on HiSeq 2500 (Illumina) rapid run flow cells with an average sequencing depth of 34.5 million paired-end reads. Read length was $100 \mathrm{bp}$. Reads were mapped on reference human genome (hg19/GRCh37 from UCSC genome release) using Tophat_2.0.6 algorithm with the following parameters: global alignment, no mismatch in seed alignment (of size 22), three mismatches in read length. Quality control was performed using FastQC software and duplicates were removed using Samtools rmdup. Gene expression quantification was performed using HTSeq-count and featureCounts (implemented in Bioconductor R package Rsubread (1.18.0)). Only genes with at least one read in $5 \%$ of all samples were kept for further analyses. Normalization was performed with DESeq2 R package (1.8.2) method. Analysis strategy includes unsupervised analyses such as PCAs and HC, as well as paired differential expression analyses (done with DESeq2 bioconductor package). Biological interpretation of the identified genes was done by computational functional analyses on Metascape (http://metascape.org), which is based on several bioinformatics resources (Gene Ontology, KEGG, Reactome). RNAseq data have been deposited on EGA website (EGA number: EGAS00001003238).

Cell culture. The human BC cell lines MCF-7(-GFP), T47D(-GFP) and MDA-MB231-GFP were propagated in DMEM (GE, \#SH30243.01) supplemented with 10\% fetal bovine serum (FBS) (Biosera, \#1003/500), penicillin (100 U/ml) and streptomycin $(100 \mu \mathrm{g} / \mathrm{ml}$ ) (Gibco \#15140122). Each cell line identity was verified by Short Tandem Repeat (STR) DNA profiling (Promega \#B9510).

CAF-S1 and CAF-S4 primary cultures were established from PT and LN human samples. All along the duration of the project, we isolated $22 \mathrm{CAF}-\mathrm{S} 1$ and $22 \mathrm{CAF}-$ S4 paired primary cell lines (referred to as CAF pairs because isolated from the same patient) using FACS-sorted cells full-filling marker criteria shown in Fig. 1. Among them, 64\% were from PTs and 36\% from LNs. In agreement with the strong transcriptomic analogy of CAF subsets from PT or LN (shown Fig. 3), we did not observe any difference in functional assays when we performed experiments with CAF-S1 or CAF-S4 isolated either from PT or from LN. Thus, we observed no effect of the site of isolation (PT or LN) on CAF-S1/CAF-S4 properties. Transcriptomic signatures and in vitro properties were thus equivalent whatever the site of CAF initial localization (PT or LN). Although CAF-S1 were quite easily expanded in vitro, CAF-S4 were difficult to maintain. As they exhibit a pericyte-like signature, we adapted the culture conditions to expand and maintain both CAF-S1 and CAF-S4 in similar and comparable conditions. BC samples were processed and sorted after surface staining as described above (see 'Flow Cytometry on BC PT and LN samples') by cell sorting and gating strategy using FACS shown in Fig. 1. Purified CAF-S1 and CAF-S4 were then plated in 96-well plates and expanded in a pericyte medium (Sciencell, \#1201) in a humidified, $1.5 \% \mathrm{O}_{2}$ and $5 \%$ $\mathrm{CO}_{2}$ incubator. Doubling time was assessed during this expansion phase for six CAF pairs over five passages. All cultures were systematically validated by qRTPCR between passages 3 and 6 using genes upregulated in either CAF-S1 or CAFS4 (including SFRP2, HMCN1, TINAGL1, PDE3A). Other types of validation were done, such as RNAseq on four CAF-S1 and CAF-S4 pairs and FACS analyses (see 'Flow Cytometry on BC PT and LN samples') to assess CAF marker protein levels (as shown in Supplementary Fig. 3). For RNAseq experiment, cells at $80 \%$ confluency were collected after trypsin treatment, total RNA was extracted (Qiagen \#217004) and DNAse-treated (Life technologies \#EN0525). mRNAs were retrotranscribed, amplified and cDNA libraries were obtained with TruSeq Stranded 
mRNA library prep (Illumina \#RS-122-2101). Samples were then sequenced and analyzed as PT and LN fresh CAF samples (see 'Gene expression profiling by RNAseq'). To validate their identity, PCA and HC were performed using CAF-S1 and CAF-S4 top-500 gene signatures. These signatures were defined as the 500 genes upregulated and the 500 genes downregulated between PT + LN CAF-S1 $(n=10)$ and CAF-S4 $(n=10)$ fresh paired samples (paired differential analysis by DESeq2). Twenty-two different CAF-S1 and CAF-S4 paired primary cell lines were isolated from BC PTs $(n=14)$ and LNs $(n=8)$ (see Supplementary Table 1 for clinical information) were used for functional experiments. All experiments were performed in DMEM with $10 \% \mathrm{FBS}$ at $1.5 \% \mathrm{O}_{2}$ unless otherwise specified. For BC cells and primary CAF cell lines, the absence of mycoplasma contamination has been tested and confirmed.

Silencing of CXCL12, CXCR4 and transient transfection. For the short interfering RNA (siRNA) experiment, CAF-S1, CAF-S4 or breast cancer cells were transfected with $20 \mathrm{nM}$ siRNA. Control was non-targeting siRNA (siCTR, AllStars negative control, \#1027281), CXCL12 silencing was performed with a siRNA pool targeting both $\alpha$ - and $\beta$-CXCL12 isoforms (Dharmacon, \#L-007873-00) and CXCR4 silencing was achieved with a pool of four specific siRNA (Dharmacon, \#L-005139-00). Transfections were performed with DharmaFECT 1 (Dharmacon, \#T-2001-02) transfection reagent according to manufacturer's instructions.

qRT-PCR from CAF subsets and BC cell lines. For gene expression analysis, total RNA isolation was performed using miRNEasy kit (Qiagen, \#217004) according to manufacturer's instructions. RNA concentrations were determined using a NanoDrop apparatus (NanoDrop Technologies, Inc.). For each sample, $1 \mu \mathrm{g}$ of total RNA was reverse transcribed using an iScript Reverse Transcription Kit (BioRad \#1708840). qRT-PCR was performed using Power SYBR Green PCR Master Mix (Applied Biosystems, \#4367659) on a CFX96 or CFX384 Touch Real-Time PCR Detection System (Bio-Rad) with primers at $300 \mathrm{nM}$ final concentration. Primers (forward and reverse) used for quantitative (q)RT-PCR amplification were: CXCL12: 5'-CTACAGATGCCCATGCCGAT-3' 5'-CAGCCGGGCTAC AATCTGAA-3', CXCR4: 5'-TGGCCTTATCCTGCCTGGTATTGT-3' ${ }^{\prime}$-AGG AGTCGATGCTGATCCCAATGT-3'. Silencing validation was performed from at least four independent experiments.

Transwell assay. Twelve-well cell culture inserts (Corning, $8 \mu \mathrm{m}$ pore size, \#353182) were used for migration assays, and migration was assessed $24 \mathrm{~h}$ after plating.

For CAF migration, after $24 \mathrm{~h}$ serum deprivation $(0.5 \% \mathrm{FBS}), 4 \times 10^{4}$ cells were plated in the upper side of the Transwell device in $0.5 \%$ FBS medium. Six CAF-S1 and CAF-S4 pairs were tested in four independent experiments, in duplicates.

For CAF chemo-attraction experiments, $10^{5} \mathrm{CAF}$ (CAF-S1 and CAF-S4) were plated in duplicates in 12-well plates. After CAF attachment $(6 \mathrm{~h})$, cells were gently washed with PBS and medium changed to $0.5 \%$ FBS. In parallel, MCF7 and T47D that had been serum deprived $(0.5 \% \mathrm{FBS})$ for $24 \mathrm{~h}$ were collected and $10^{5}$ cells were plated in the upper side of the Transwell device in $0.5 \%$ FBS medium, on top of $0.5 \%$ FBS medium \pm CAF. Representative images of Transwell membrane underside shown in Fig. 4 are obtained $24 \mathrm{~h}$ after CAF-S1/CAF-S4 seeding in upper part. At least eight independent experiments including at least four different CAFS1 and CAF-S4 pairs were performed with each BC cell line, each condition in duplicates.

To assess CXCL12 involvement in CAF-S1 attraction of BC cells, $10^{5}$ CAF-S1 were plated as described above, after $48 \mathrm{~h}$ of transient silencing (see 'Silencing of CXCL12, CXCR4 and transient transfection'). BC cell number was increased to $3 \times$ $10^{5}$ cells per insert and plated in triplicates. Three independent experiments including at least two different CAF-S1 cell lines per BC cell type were performed.

At the end of the experiment, the remaining cells in the upper side of the Transwell device were gently removed with cotton swabs moistened with PBS. Migrating cells at the bottom side of the Transwell device were fixed and stained with crystal violet for $30 \mathrm{~min}$ and then counted in seven different representative fields per insert (X5 objective, Zeiss Axioplan microscope, AxioCamERc 5s, manual counting on Fiji software) and averaged per condition. When we adjusted the migration to proliferation rate, we divided the migration capacity by the fold change in tumor cell proliferation in presence/absence of CM from CAF subsets.

Cell exclusion zone assay. For cell exclusion zone assay, Ibidi culture inserts (\#80209) were added to 12 -well plates (one insert per well) and $10^{4}$ CAF-S1 or $10^{4}$ CAF-S4 were plated per chamber of each insert, in duplicates. After cell attachment, inserts were gently removed, delicately washed with PBS and $2 \mathrm{ml}$ of medium was added to each well. Time-lapse videos were then immediately acquired with an inverted motorized Leica video-microscopes, equipped with motorized stage, $37^{\circ} \mathrm{C}$ incubator and $\mathrm{CO}_{2}$ controller (Leica DMi8 with a Retiga R6 camera and illumination by Lumencor SOLA SE 365). The automated imaging system was controlled by the software Metamorph (Universal Imaging). Transmission images were acquired every $15 \mathrm{~min}$ until $50 \mathrm{~h}$ and one position per insert (hence two per condition) was taken with a $5 \times$ objective as it encompassed $80 \%$ of the cell-free zone. For data analyses, Fiji software was used. In each position, manual tracking of $40 \mathrm{CAF}-\mathrm{S} 1$ and CAF-S4 cells per condition positioned on the free edges were tracked over $24 \mathrm{~h}$, taking into consideration the first image of each hour. Velocity (distance of migration divided by the interval time) and direction (absolute value of $\sin (\alpha)$, where $\alpha$ is the angle between the velocity and the initial cell-free zone border - absolute because cells were taken from both sides of the cell-free zone) were calculated for each successive pair of time points for each cell. Means per cell over the global tracking are shown. Persistence of cell trajectory, defined as the ratio $d / l$ where $l$ is the contour length of the total trajectory and $d$ the direct endto-end distance, was also calculated per cell over the same $24 \mathrm{~h}$. Two different CAF$\mathrm{S} 1$ and CAF-S4 pairs were tested in two independent experiments.

CAF spheroids and invasion assay. For spheroid invasion assays, CAFs were trypsinized and resuspended at a concentration of $4 \times 10^{5}$ cells $/ \mathrm{ml}$. Atotal of $20 \mu \mathrm{l}$ drops were pipetted down on a $10-\mathrm{cm}$ dish lid. Lids were then gently put back on the plates filled with medium to humidify the system. After 4 days, CAF spheroids -formed in the hanging drops-were carefully transferred into $2.5 \mathrm{mg} / \mathrm{ml}$ collagen solution drops (Corning, \#354249) in the center of 6-well plates. At least six different spheroids per CAF were embedded in collagen and further analyzed. After polymerization, $0.5 \%$ FBS medium was added to the wells. Spheroids were imaged 3 days after inclusion on a Nikon Eclipse TE300 microscope with a $4 \times$ objective equipped with a Nikon DS-Fil camera and invasion was assessed with Fiji software as an invasion index defined as the total area covered by CAFs (encompassing single cells escaped from the spheroid and the spheroid) divided by the area of the spheroid core center. Three CAF-S1 and CAF-S4 pairs were tested in three independent experiments. For time-lapse imaging, time-lapse videos were immediately acquired after collagen polymerization with an inverted motorized Leica videomicroscopes, equipped with motorized stage, $37^{\circ} \mathrm{C}$ incubator and $\mathrm{CO}_{2}$ controller (Leica DMi8 with a Retiga R6 camera and illumination by Lumencor SOLA SE 365). The automated imaging system was controlled by the software Metamorph (Universal Imaging). Transmission images were acquired every 15 min until $42 \mathrm{~h}$ with a $5 \times$ objective to encompass the full spheroid. Videos were mounted with eight frames per second.

Collagen contraction assay. To assess force-mediated collagen contraction, $3 \times$ $10^{4} \mathrm{CAFs}$ were embedded in $100 \mu \mathrm{l}$ of a $2.5-\mathrm{mg} / \mathrm{ml}$ collagen solution (Corning, \#354249) and seeded in triplicates in 96-well plates. After polymerization, culture medium was added on top of the gels and in the surrounding empty wells to limit evaporation. To assess DAPT (Sigma, \#D5942), BLEBBISTATIN (Sigma, \#B0560) and Y27632 (STEMCELL Technologies, \#72302) impact on CAF contractility, $10 \mu \mathrm{M}$ of each drug was added to the medium, DMSO as control. Gel contraction was assessed 4 days after plating: plates were scanned (EPSON Perfection V700 Photo scanner) and quantification was performed on Fiji software. Percentage of contraction was calculated using the formula:

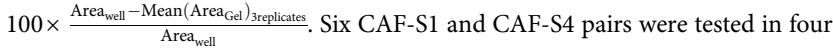
independent experiments. For DAPT experiments, three independent experiments were performed, including four different CAF-S4 cell lines.

Traction force microscopy. For TFM experiments, $2.5 \times 10^{4} \mathrm{CAFs}$ were seeded on collagen pre-coated $25 \mathrm{kPa}$ hydrogels containing fluorescent beads (Matrigen, \#ST0.2YG-SW6G-COL-25 EA). These commercial hydrogels contain fluorescent beads of $0.2 \mu \mathrm{m}$ diameter at a density of $0.2 \pm 0.04$ beads $/ \mu \mathrm{m}^{2}$. Three CAF-S1 and CAF-S4 pairs were tested. Cells were plated at low density $\left(\sim 25\right.$ cells $\left./ \mathrm{mm}^{2}\right)$ to ensure that traction forces were measured on single cells. When DAPT (Sigma, \#D5942) treatment was evaluated, $10 \mu \mathrm{M}$ was added to the medium, DMSO as control. Gels (transmission and GFP channels) were imaged on an inverted Leica videomicroscope (Leica DMi8 with a Retiga R6 camera and illumination by Lumencor SOLA SE 365) $24 \mathrm{~h}$ post-plating with $20 \times$ objective, the large size of CAF cells with elongated protrusions precluding the use of higher magnification. Images of the relaxed gels were acquired after removing the cells with trypsin. Beads images (with and without the cells) were first aligned using the ImageJ plugin "Align slices in stack".

Bead displacements (with respect to the resting state after trypsination) were measured and converted to cell traction stresses using the open source Fourier transform traction cytometry Fiji plugins (available at https://sites.google.com/site/ qingzongtseng/tfm or org.insb.bib.cnrs.fr/10.1016/bs.mcb.2014.10.008)

Images were processed with ImageJ software. Further analyses were performed with Matlab (MathWorks, Natick, MA). To quantify cell contractility, we measured the strain energy (SE) which is the total energy transferred from the cell to the elastic substrate, the strain energy density which is the energy per unit area (SE divided by the cell area) and the mean traction stress amplitude which corresponds to the average stress per cell. SE $=\frac{1}{2} \int_{\text {Cell }} \vec{T}(\vec{r}) \cdot \vec{u}(\vec{r}) d^{2} \vec{r}$,

where $\vec{T}$ is the traction stress field and $\vec{u}()$ is the displacement field.

\section{Collagen evaluation by second harmonic generation. CAFs were seeded in} home-made holes in $30 \mathrm{~mm}^{2}$ tissue culture plates as described in ref. ${ }^{23}$ : three holes of 3-4 mm diameter were drilled in a plate and widened around the edges using a scalpel. The bottom of the dish was covered with epoxy (Loctite), and $20 \times 20 \mathrm{~mm}$ square coverslips were glued to the dish overnight at RT. The day before the experiment, dishes were silanized with 3-aminopropyl-trimethoxysilane (Sigma- 
Aldrich) and extensively washed with water treated for $30 \mathrm{~min}$ with $0.5 \%$ glutaraldehyde followed by a final wash, to avoid collagen detachment from the plastic holes caused by CAF contractility. A total of 3000 CAF-2 CAF-S1 and CAF-S4 pairs were tested-were embedded in $15 \mu \mathrm{l}$ of a $2.5-\mathrm{mg} / \mathrm{ml}$ collagen solution (Corning, \#354249) and seeded in duplicates in the afore-mentioned holes. After polymerization, medium was added to the plates. Cells were left 3 days in this system, then fixed for $30 \mathrm{~min}$ in $4 \%$ PFA, permeabilized with $0.1 \%$ Triton X-100 in PBS for $30 \mathrm{~min}$ and F-actin was stained using phalloidin (Thermo Fisher Scientific, \#R415, 1:200 O/N in PBS at $4^{\circ} \mathrm{C}$ ). Images (of at least 10 cells per CAF over two wells) were acquired with an inverted AOBS two-photon laser-scanning confocal microscope (SP8; Leica) coupled with a femtosecond laser (Chameleon Vision II; Coherent Inc.) using a $25 \times / 1.0 \mathrm{NA}$ water immersion objective. The microscope was equipped with three nondescanned HyD detectors: NDD1 (500-550 nm), NDD2 $(\geq 590 \mathrm{~nm})$ and NDD3 $(405 \mathrm{~nm})$. Fluorescence channel was recorded using the excitation wavelength $561 \mathrm{~nm}$. Collagen was visualized by second harmonic generation (SHG) using the excitation wavelength $910 \mathrm{~nm}$. 3D stacks were acquired at a step size of $1 \mu \mathrm{m}$ intervals to encompass one whole cell. Images were further analyzed with Fiji software. All collagen images were processed in the same way: light was adjusted $(\min =0$ and $\max =50)$, thresholded $(\min =0, \max =30)$, converted to mask, smoothed, despeckled and collagen area was measured on all slices of each 3D-stack. Collagen density around each cell was then computed as $d=\frac{\left.\sum_{i=1}^{n} \Delta z \times \text { surf.coll }_{\text {sice(i) }}\right)}{V_{\text {sank }}}$, where $\Delta z$ is the step between two slices in the stack, $n$ the number of slices in the stack, $V_{\text {stack }}$ the volume of the stack and surf.coll slice(s) $_{\text {s }}$ the area covered by collagen in the slice $i$ of the stack (Fiji output).

Proliferation assays. CAF doubling time was assessed over five passages for six pairs or CAF-S1 and CAF-S4 primary cell lines, CAFs being sub cultured when reaching $90 \%$ confluency in the plate (see 'Cell culture').

For co-culture proliferation assay, $10^{5} \mathrm{CAF}(-\mathrm{S} 1$ or $-\mathrm{S} 4)$ and/or $5 \times 10^{4} \mathrm{MCF} 7$ cells $\left(1.5 \times 10^{5} \mathrm{CAF}(-\mathrm{S} 1\right.$ or $-\mathrm{S} 4)$ and/or $7.5 \times 10^{4} \mathrm{~T} 47 \mathrm{D}$ cells) were plated in duplicates in 6-well plates. After cell attachment, cells were washed in PBS and $0.5 \%$ medium was added; $24 \mathrm{~h}$ or $72 \mathrm{~h}$ later, cells were collected (cells in supernatant as well), washed with PBS+ and stained for $15 \mathrm{~min}$ at RT with antiEpCAM-BV605 (BioLegend, \#324224) and anti-FAP-APC (primary antibody, R\&D Systems, \#MAB3715 conjugated with fluorescent dye Zenon APC Mouse IgG1 labeling kit, Thermo Fisher Scientific, \#Z25051) to distinguish CAFs from BC cells. After a last washing step, all samples were resuspended in $50 \mu \mathrm{l}$ of PBS+ containing $2.5 \mu \mathrm{g} / \mathrm{ml}$ DAPI (Thermo Fisher scientific, \#D1306) and 1:100 carboxylated beads (Polyscience, \#18133). Total samples were acquired on the LSRFortessa ${ }^{\mathrm{TM}}$ analyzer (BD biosciences) and precision beads were used to normalize viable $\mathrm{BC}$ cell $\left(\mathrm{DAPI}^{-} \mathrm{EPCAM}^{+} \mathrm{FAP}^{-}\right)$and $\mathrm{CAF}\left(\mathrm{DAPI}^{-} \mathrm{EPCAM}^{-}\right.$ $\mathrm{FAP}^{+}$) counts. At least six independent experiments including at least three different CAF-S1 and CAF-S4 pairs were performed per BC cell line.

For BC cell viability assessment with CAF CM (three CAF pairs for each three independent experiments and four different CAF-S1 in two independent experiments for CXCL12 silencing), as well as CAF viability assessment with DAPT, BLEBBISTATIN (Sigma, \#B0560) or Y27632 (STEMCELL Technologies, \#72302) treatment (three independent experiments with two different CAF-S4 each time), we used resazurin assay. Briefly, $10^{4} \mathrm{BC}$ cells were seeded in triplicates in 96well plates. To evaluate CM-CAF effect, once $\mathrm{BC}$ cells attached, cells were gently washed with PBS and $100 \mu \mathrm{l}$ of CAF-CM were added to each well. Cell viability was assayed $24 \mathrm{~h}$ later. To obtain CM-CAF, $10^{5} \mathrm{CAF}$ were plated in 12 -well plates in duplicates (24 h post siRNA transfection for CXCL12 silencing). After attachment, cells were gently washed with PBS and $1 \mathrm{ml}$ of $0.5 \%$ FBS medium was added per well. After $24 \mathrm{~h}$, supernatants were collected, duplicates were mixed, centrifugated and immediately used for experiments. For CAF viability assessment, $7 \times 10^{3} \mathrm{CAFs}$ were seeded in triplicates in 96-well plate wells with the different drugs at $10 \mu \mathrm{M}$ in the medium (DMSO was used as control). Cell viability was assessed after $24 \mathrm{~h}$, $72 \mathrm{~h}$ and 7 days. To do so, $25 \mu \mathrm{l}$ of resazurin reagent $(0.05 \mathrm{mg} / \mathrm{ml}$, Sigma \#R7017) was added to each well. Plates were incubated at $37^{\circ} \mathrm{C}$ for $4 \mathrm{~h}$ and read in a Multi Detection plate reader (Fluostar, BMG Labtech).

Immunofluorescence. For immunofluorescence experiments, 200,000 BC cells \pm 200,000 CAF (24 h post-silencing when CXCL12 and CXCR4 roles in EMTtriggering was assessed) were seeded on coverslips placed in 6-well plate wells. When TGF $\beta$-R involvement was assessed in EMT-triggering, the TGF $\beta$-RI/II inhibitor LY2109761 (Selleckchem, \#S2704) was added at $5 \mu \mathrm{M}$ in the medium (DMSO was used as control). Forty-eight hours later, cells were fixed in 4\% PFA for $15 \mathrm{~min}$, permeabilized with $0.1 \%$ SDS in PBS for $10 \mathrm{~min}$, blocked in PBS-Tween $0.1 \%$ with 5\% BSA (Euromedex, \#04-100-812-C) for $30 \mathrm{~min}$ and incubated with antibodies diluted in PBS-Tween $0.1 \%$ with $5 \%$ BSA overnight at $4{ }^{\circ} \mathrm{C}$. Antibodies were anti-Vinculin (1:1000, Sigma, \#V9131) or E-cadherin (1:300, Cell Signaling Technology, \#3195). Cells were then incubated with Cy3-anti-mouse secondary (1:500, JacksonImmunoResearch, \#715-165-150) or Cy3-anti-rabbit secondary (1:500, JacksonImmunoResearch, \#711-165-152) in parallel with Alexa Fluor TM 488 phalloidin (1:200, Invitrogen, \#A12379) for $30 \mathrm{~min}$ at RT in PBS-Tween $0.1 \%$ with $5 \%$ BSA. After several washing steps, coverslips were mounted on slides with a drop of Vectashield mounting medium with DAPI (Vector, \#H-1200). Slides were then examined using an upright Epifluorescence Microscope with Apotome (Zeiss) with a $40 \times$ oil-immersion objective and images (at least five positions per condition) were acquired with identical exposure times and settings using a digital camera (Photometrics CoolSNAP HQ2). At least two independent experiments were performed for each setting and each BC cell line (one CAF pair for CAF-S1 versus CAF-S4 impact in two independent experiments; three CAF-S1 in three independent experiments for CXCL12 involvement; two CAF-S1 in two independent experiments for CXCR4 involvement; at least three CAF-S1 in at least three independent experiments for TGF $\beta-R$ involvement).

Tumor cell density, E-cadherin in BC cells, F-actin and Vinculin in CAF. For tumor cell density and assessment of staining in CAF subsets, quantifications were performed in two steps on Fiji software. (1) Masks were manually drawn on each merged image to either keep tumor cell zones of CAF zones according to the analyses. Masks were then applied to the corresponding single channel (Dapi, Ecadherin, F-actin or Vinculin depending on the analyses) images. (2) For BC cell density, evaluation of cell number was automatically performed by applying a threshold filter $(0,20)$ on Dapi channel (raw images opened and auto-scaled with Bio-formats plug-in, saved in jpeg format) followed by a mask conversion, segmentation and then particles were analyzed (size 2000-25,000; circularity $>0.2$ ). Cell number was then divided by the tumor zone area. For F-actin or Vinculin signal extraction in CAF, corresponding images were thresholded (a single threshold was set up per experiment on raw images opened and auto-scaled with Bio-formats plug-in, saved in jpeg format: 125 for F-actin, 150-175 for Vinculin), converted to masks, integrated density was retrieved and then divided by CAF zone area. For E-cadherin signal extraction, as CAFs were negative for E-cadherin, step (1) was skipped and step (2) was directly performed. E-cadherin signal area shown in Figs. 5, 7 was defined on ImageJ, and next divided by tumor cell surface. Images were thresholded (single threshold per experiment ranging from 50 to 225 applied on raw images opened and auto-scaled with Bio-formats plug-in, saved in jpeg format), converted to masks, integrated density was retrieved and then divided by tumor zone area.

Inverted Transwell assay. For inverted Transwell assays, CAF were first labeled with fluorescent dyes (Thermo Fisher Scientific, for CAF-S1: CellTrace Yellow \#C34573, for CAF-S4: CellTrace Violet, \#C34571) according to manufacturer's protocol; $2 \times 10^{4}$ cells were then embedded in $100 \mu \mathrm{l}$ of a $2.5-\mathrm{mg} / \mathrm{ml}$ collagen solution (Corning, \#354249) and seeded inside 24-well cell culture inserts (Corning, $8 \mu \mathrm{m}$ pore size, \#3422). CAF-free collagen was used as control. After polymerization, inserts were put upside-down on plate lids and $4 \times 10^{5} \mathrm{MDA}-\mathrm{MD}-231$ GFP were seeded on the bottom part of the inserts and left to adhere for $2 \mathrm{~h}$. Inserts were then placed in 24 -well plates containing $0.5 \%$ FBS and complete medium was added inside the insert plates. After $24 \mathrm{~h}$, inserts were gently transferred into new 24-well plates containing 0.5\% FBS and further incubated for 6 days. All conditions were done in duplicates. After 7 days of invasion, inserts were gently washed in PBS, fixed in 4\% PFA for 15 min, washed again and mounted with Mounting medium (Aqua-Poly/Mount \#18606) on glass-bottom dishes (FisherScientific \#15159112). Images were then acquired on an inverted Laser Scanning Confocal Microscope with Spectral Detection and Multi-photon Laser (LSM880NLO/Mai Tai Laser-Zeiss/Spectra Physics) using a 25×/0.8 NA oil immersion objective. 3D stacks were acquired at a step size of $10 \mu \mathrm{m}$ intervals to encompass all invading cells (initial $Z$ set up so that membrane pores were in focus and highest $Z$ where no MDA-MB-231-GFP cells could be detected anymore). Fluorescence channels were recorded using the excitation wavelengths $405 \mathrm{~nm}$ (for CAF-S4), $488 \mathrm{~nm}$ (for BC cells) and $561 \mathrm{~nm}$ (for CAF-S1). At least four stacks were acquired per insert, $x$ and $y=566.8 \mu \mathrm{m} ; z=200 \mu \mathrm{m}$. Images were further analyzed with IMARIS software to retrieve the distance of invasion of all BC cells in each stack, after modeling cells on GFP signals done with the same parameters across stacks (surface detail $2 \mu \mathrm{m}$; threshold absolute intensity 3.00; option "enable splitting of touching objects" was selected; seed points diameter $15 \mu \mathrm{m}$; filtered on voxels above 350 ). Each stack was normalized to the number of detected cells in the stack to express frequencies of cells. Maximal distance of invasion was defined as the value of $Z$ above which $1 \%$ of cells in stack was found. Data were then averaged per condition per experiment. Three independent experiments with two different CAF-S4 cell lines were performed.

For DAPT (Sigma, \#D5942) treatment, when CAF-S4 were used, they were labeled and included in collagen as described above. MDA-MB-231-GFP cells were seeded as described above (below CAF-S4-free collagen for control experiments or CAF-S4-embedded collagen). $10 \mu \mathrm{M}$ DAPT or DMSO was added on both sides of the inserts and media were changed at day 3 . All conditions were done in duplicates. Inserts were fixed, acquisition and analysis were performed as described above with the following Imaris parameters: surface detail $2 \mu \mathrm{m}$; Threshold absolute intensity 2.00; option "enable splitting of touching objects" was selected; seed points diameter $15 \mu \mathrm{m}$; filtered on voxels above 300. 5 independent control experiments were performed and 6 independent experiments including 5 different CAF-S4 cell lines were performed to assess DAPT effect on CAF-S4-induced invasion of $\mathrm{BC}$ cells.

Tumor-on-chip assay. The devices were made of PDMS (polydimethylsiloxane), a biocompatible, gas-permeable and transparent silicone rubber. The devices were 
micro-fabricated at the Institut Pierre-Gilles de Gennes pour la Microfluidique (IPGG, Paris) using standard soft-lithography or advanced micro-milling for the mold fabrication and soft-lithography methods to prepare the chips. The microfluidic device were sterilized in UV-oven ( $365 \mathrm{~nm}$ wavelength) for $30 \mathrm{~min}$, coated with $10 \mu \mathrm{g} / \mathrm{ml}$ human fibronectin (Sigma, \#F0895) overnight at $37^{\circ} \mathrm{C}, 5 \% \mathrm{CO}_{2}$, rinsed with PBS and then dried for at least $5 \mathrm{~h}$ in a sterile room. Cells were embedded in $2.3 \mathrm{mg} / \mathrm{ml}$ collagen hydrogels (PureCol, Advanced Matrix, \# 5005 diluted in MEM medium (Sigma Aldrich, \# M0275) containing 0.28\% $\mathrm{NaHCO}_{3}$ $(\mathrm{pH}=8)$ to adjust the final $\mathrm{pH}$ to 7$)$. MCF7-GFP or T47D-GFP targeted density was $1.2 \times 10^{6}$ cells $/ \mathrm{ml}$, CAF density was $3 \times 10^{5}$ cells $/ \mathrm{ml}$. When DAPT (Sigma, \#D5942) was used, CAF-S4 were pre-treated overnight before inclusion in collagen and a final concentration of $10 \mu \mathrm{M}$ was added to the medium. We verified that DMSO did not interfere with BC cell velocity in the system. Time-lapse videos were then acquired with an inverted motorized Leica video-microscopes, equipped with motorized stage, $37^{\circ} \mathrm{C}$ incubator and $\mathrm{CO}_{2}$ controller (Leica DMi8 with a Retiga R6 camera and illumination by Lumencor SOLA SE 365) in a $20 \% \mathrm{O}_{2}$ environment. The automated imaging system was controlled by the software Metamorph (Universal Imaging). Transmission and fluorescent (GFP) images were acquired every $2 \mathrm{~h}$ over $30 \mathrm{~h}$ and at least four positions per condition were recorded with a $10 \times$ objective. For data analyses, Fiji software was used. In each position, cells were manually tracked over all frames ('Manual tracking' plugin, at least 65 cells per condition). Cell velocity (distance of migration divided by the interval time) was calculated for each successive pair of time points. Means per cell over the global tracking are shown. For CAF-S1 versus CAF-S4 experiments, for each BC cell line: three independent experiments were performed, with three different CAF-S1 and CAF-S4 pairs. For DAPT experiments, for each BC cell lines: three independent experiments were performed, including two different CAF-S4 cell lines.

Cytokine antibody-pair-based assay. Cytokine antibody-pair-based assays were performed using human cytokine array kit (R\&D Systems, \#ARY005B) according to the manufacturer's protocol. In brief, 80,000 CAF-S1 or CAF-S4 fibroblasts were cultured alone or in presence of $80,000 \mathrm{BC}$ cells (MCF7 or T47D) in $1 \mathrm{ml}$ of DMEM medium in 12 -well plates at $1.5 \% \mathrm{O}_{2}$ during $48 \mathrm{~h}$. Culture supernatants were next collected (CM) and centrifuged at 13,000 rpm during $10 \mathrm{~min}$ for eliminating debris. Array membranes, previously spotted with capture antibodies by the manufacturer, were incubated with $0.5 \mathrm{ml}$ of $\mathrm{CM}$ overnight at $4{ }^{\circ} \mathrm{C}$. Membranes were then washed three times with $50 \mathrm{ml}$ of washing buffer at RT, and incubated with a streptavidin-horseradish peroxidase-coupled antibody (1:2000) for $30 \mathrm{~min}$ at RT and revealed using Chemi-Reagent Mix. The immunoblot images were captured and visualized using the ChemiDoc Imaging System (Bio-Rad) and intensity of each spot in the captured images was measured using ImageJ software. Two spots per membrane were used to measure the levels of each cytokine. Intensity mean of the two spots-revealing each cytokine level per experimentwas reported to the intensity mean of three internal controls spotted on the membrane and assessed by the following ratio: specific intensity mean / control intensity mean, to take into consideration variation in hybridization efficiency in the different experiments.

Software. Software used for the above analyses are: R (https://cran.r-project.org, R versions 3.3.1, 3.4.0 and 3.5.0), Fiji (ImageJ v2.0.0-rc-19/1.49m, v2.0.0-rc-65/1.52b), IMARIS (v8.4.1), Matlab.

Statistical analyses. Data graphical representation and statistical analyses were done using R or GraphPad Prism environment. Barplots represent means \pm stan dard errors of the mean (SEM). Boxplots: box limits indicate the inter-quartile range (IQR) (25th to 75 th percentiles), with a center line indicating the median. Whiskers show value ranges up to $1.5 \times \mathrm{IQR}$ above the 75 th or below the 25 th percentiles, with outliers beyond those ranges shown as individual points. The number of independent experiments is specified in each figure legend, with at least three independent experiments, unless otherwise specified. The statistical tests used are in agreement with the data distribution: normality was first checked using the Shapiro-Wilk test and parametric or non-parametric two-tailed tests were applied according to normality. Statistical test types are indicated in figure legends. Survival analyses were carried out with survival R package by Kaplan-Meier curves ( $p$ values from Log-rank test) and by univariate and multivariate Cox regressions using additive hazards regression models. Stratification of patients for Kaplan-Meier analyses were performed using median value of the stromal content distribution and CAF-S1 or CAF-S4 enrichments (defined by our algorithm, see 'IHC analyses'). Differences were considered statistically significant when $p \leq 0.05$.

Reporting summary. Further information on research design is available in the Nature Research Reporting Summary linked to this article.

\section{Data availability}

RNAseq data from $\mathrm{EPCAM}^{+}$, CAF-S1 and CAF-S4 sorted from BC samples, without or with culture, generated in this study are available on European Genome-Phenome Archive platform (https://ega-archive.org) under accession number: EGAS00001003238.
A source data file corresponding to all panels of the figures and supplementary figures that support the findings of this study is provided with the paper.

\section{Code availability}

$\mathrm{R}$ scripts used to generate panels of the figures and supplementary figures are provided within the data source file of the paper, available at https://doi.org/10.6084/m9. figshare.9924275.

Received: 13 February 2019; Accepted: 17 December 2019; Published online: 21 January 2020

\section{References}

1. Kast, K. et al. Impact of breast cancer subtypes and patterns of metastasis on outcome. Breast Cancer Res. Treat. 150, 621-629 (2015).

2. $\mathrm{Wu}, \mathrm{Q}$. et al. Breast cancer subtypes predict the preferential site of distant metastases: a SEER based study. Oncotarget 8, 27990-27996 (2017).

3. Tang, C. et al. Lymph node status have a prognostic impact in breast cancer patients with distant metastasis. PLoS ONE 12, e0182953 (2017).

4. Puram, S. V. et al. Single-cell transcriptomic analysis of primary and metastatic tumor ecosystems in head and neck cancer. Cell 171, 1611-1624. e1624 (2017).

5. Brabletz, T., Kalluri, R., Nieto, M. A. \& Weinberg, R. A. EMT in cancer. Nat Rev. Cancer 18, 128-134 (2018).

6. Puisieux, A., Pommier, R. M., Morel, A. P. \& Lavial, F. Cellular Pliancy and the multistep process of tumorigenesis. Cancer Cell 33, 164-172 (2018).

7. Ombrato, L. et al. Metastatic-niche labelling reveals parenchymal cells with stem features. Nature 572, 603-608 (2019).

8. Balkwill, F. R., Capasso, M. \& Hagemann, T. The tumor microenvironment at a glance. J. Cell Sci. 125, 5591-5596 (2012).

9. Hanahan, D. \& Coussens, L. M. Accessories to the crime: functions of cells recruited to the tumor microenvironment. Cancer Cell 21, 309-322 (2012)

10. Tichet, M. et al. Tumour-derived SPARC drives vascular permeability and extravasation through endothelial VCAM1 signalling to promote metastasis. Nat. Commun. 6, 6993 (2015).

11. Gentric, G., Mieulet, V. \& Mechta-Grigoriou, F. Heterogeneity in cancer metabolism: new concepts in an old field. Antioxid. Redox Signal. 26, 462-485 (2017).

12. Kalluri, R. \& Zeisberg, M. Fibroblasts in cancer. Nat. Rev. Cancer 6, 392-401 (2006).

13. Pietras, K. \& Ostman, A. Hallmarks of cancer: interactions with the tumor stroma. Exp. Cell Res. 316, 1324-1331 (2010).

14. Costa, A., Scholer-Dahirel, A. \& Mechta-Grigoriou, F. The role of reactive oxygen species and metabolism on cancer cells and their microenvironment. Semin. Cancer Biol. 25, 23-32 (2014).

15. Kalluri, R. The biology and function of fibroblasts in cancer. Nat. Rev. Cancer 16, 582-598 (2016).

16. Orimo, A. et al. Stromal fibroblasts present in invasive human breast carcinomas promote tumor growth and angiogenesis through elevated SDF-1/ CXCL12 secretion. Cell 121, 335-348 (2005).

17. Toullec, A. et al. Oxidative stress promotes myofibroblast differentiation and tumour spreading. EMBO Mol. Med. 2, 211-230 (2010).

18. Attieh, Y. \& Vignjevic, D. M. The hallmarks of CAFs in cancer invasion. Eur. J. Cell Biol. 95, 493-502 (2016).

19. Erdogan, B. \& Webb, D. J. Cancer-associated fibroblasts modulate growth factor signaling and extracellular matrix remodeling to regulate tumor metastasis. Biochem. Soc. Trans. 45, 229-236 (2017).

20. LeBleu, V. S. \& Kalluri, R. A peek into cancer-associated fibroblasts: origins, functions and translational impact. Dis. Model. Mech. 11, dmm029447 (2018).

21. Labernadie, A. et al. A mechanically active heterotypic E-cadherin/N-cadherin adhesion enables fibroblasts to drive cancer cell invasion. Nat. Cell Biol. 19, 224-237 (2017)

22. Goetz, J. G. et al. Biomechanical remodeling of the microenvironment by stromal caveolin-1 favors tumor invasion and metastasis. Cell 146, 148-163 (2011).

23. Attieh, Y. et al. Cancer-associated fibroblasts lead tumor invasion through integrin-beta3-dependent fibronectin assembly. J. Cell Biol. 216, 3509-3520 (2017).

24. Gaggioli, C. et al. Fibroblast-led collective invasion of carcinoma cells with differing roles for RhoGTPases in leading and following cells. Nat. Cell Biol. 9, 1392-1400 (2007).

25. Calvo, F. et al. Mechanotransduction and YAP-dependent matrix remodelling is required for the generation and maintenance of cancer-associated fibroblasts. Nat. Cell Biol. 15, 637-646 (2013). 
26. Glentis, A. et al. Cancer-associated fibroblasts induce metalloproteaseindependent cancer cell invasion of the basement membrane. Nat. Commun. 8, 924 (2017).

27. Kim, H. M., Jung, W. H. \& Koo, J. S. Expression of cancer-associated fibroblast related proteins in metastatic breast cancer: an immunohistochemical analysis. J. Transl. Med. 13, 222 (2015).

28. Mundim, F. G. L. et al. Breast carcinoma-associated fibroblasts share similar biomarker profiles in matched lymph node metastasis. Appl. Immunohistochem. Mol. Morphol. 00, 1-9 (2016).

29. Gerstenberger, W. et al. Stromal caveolin-1 and caveolin-2 expression in primary tumors and lymph node metastases. Anal. Cell. Pathol. 2018, 1-8 (2018).

30. Niemiec, J. et al. Podoplanin-positive cancer-associated stromal fibroblasts in primary tumor and synchronous lymph node metastases of HER2overexpressing breast carcinomas. Anticancer Res. 38, 1957-1965 (2018).

31. Ohlund, D. et al. Distinct populations of inflammatory fibroblasts and myofibroblasts in pancreatic cancer. J. Exp. Med. 214, 579-596 (2017).

32. Costa, A. et al. Fibroblast heterogeneity and immunosuppressive environment in human breast cancer. Cancer Cell 33, 463-479.e410 (2018).

33. Givel, A. M. et al. miR200-regulated CXCL12beta promotes fibroblast heterogeneity and immunosuppression in ovarian cancers. Nat. Commun. 9, 1056 (2018).

34. Su, S. et al. CD10(+)GPR77(+) cancer-associated fibroblasts promote cancer formation and chemoresistance by sustaining cancer stemness. Cell 172, 841-856.e816 (2018)

35. Van Gassen, S. et al. FlowSOM: using self-organizing maps for visualization and interpretation of cytometry data. Cytometry A 87, 636-645 (2015).

36. Fletcher, A. L. et al. Reproducible isolation of lymph node stromal cells reveals site-dependent differences in fibroblastic reticular cells. Front. Immunol. 2, 35 (2011).

37. Vecchi, M. et al. Breast cancer metastases are molecularly distinct from their primary tumors. Oncogene 27, 2148-2158 (2008).

38. Hosaka, K. et al. Pericyte-fibroblast transition promotes tumor growth and metastasis. Proc. Natl Acad. Sci. USA 113, E5618-E5627 (2016).

39. Murgai, M. et al. KLF4-dependent perivascular cell plasticity mediates pre-metastatic niche formation and metastasis. Nat. Med. 23, 1176-1190 (2017).

40. Bartoschek, M. et al. Spatially and functionally distinct subclasses of breast cancer-associated fibroblasts revealed by single cell RNA sequencing. Nat. Commun. 9, 5150 (2018).

41. Di Carlo, S. E. \& Peduto, L. The perivascular origin of pathological fibroblasts. J. Clin. Investig. 128, 54-63 (2018).

42. Nguyen, M. et al. Dissecting effects of anti-cancer drugs and of cancerassociated fibroblasts by on-chip reconstitution of immunocompetent tumor microenvironments. Cell Rep. 25, 884-3893 (2019).

43. Clark, A. G. \& Vignjevic, D. M. Modes of cancer cell invasion and the role of the microenvironment. Curr. Opin. Cell Biol. 36, 13-22 (2015).

44. Yamashita, M. et al. Role of stromal myofibroblasts in invasive breast cancer: stromal expression of alpha-smooth muscle actin correlates with worse clinical outcome. Breast Cancer 19, 170-176 (2012).

45. Paulsson, J. \& Micke, P. Prognostic relevance of cancer-associated fibroblasts in human cancer. Semin. Cancer Biol. 25, 61-68 (2014).

46. Qiao, A., Gu, F., Guo, X., Zhang, X. \& Fu, L. Breast cancer-associated fibroblasts: their roles in tumor initiation, progression and clinical applications. Front. Med. 10, 33-40 (2016).

47. Sjoberg, E., Augsten, M., Bergh, J., Jirstrom, K. \& Ostman, A. Expression of the chemokine CXCL14 in the tumour stroma is an independent marker of survival in breast cancer. Br. J. Cancer 114, 1117-1124 (2016).

48. Amornsupak, K. et al. High $\operatorname{ASMA}(+)$ fibroblasts and low cytoplasmic HMGB1 $(+)$ breast cancer cells predict poor prognosis. Clin. Breast Cancer 17, 441-452.e442 (2017)

49. Vangangelt, K. M. H. et al. The prognostic value of tumor-stroma ratio in tumor-positive axillary lymph nodes of breast cancer patients. Int. J. Cancer 143, 3194-3200 (2018).

50. Calon, A., Tauriello, D. V. \& Batlle, E. TGF-beta in CAF-mediated tumor growth and metastasis. Semin. Cancer Biol. 25, 15-22 (2014).

51. Del Pozo Martin, Y. et al. Mesenchymal cancer cell-stroma crosstalk promotes niche activation, epithelial reversion, and metastatic colonization. Cell Rep. 13, 2456-2469 (2015).

52. Lo, A. et al. Fibroblast activation protein augments progression and metastasis of pancreatic ductal adenocarcinoma. JCI Insight 2, 92232 (2017).

53. Strell, C. et al. Impact of epithelial-stromal interactions on peritumoral fibroblasts in ductal carcinoma in situ. J. Natl. Cancer Inst. 111, 983-995 (2019).

54. Allinen, M. et al. Molecular characterization of the tumor microenvironment in breast cancer. Cancer Cell 6, 17-32 (2004).

55. Boimel, P. J. et al. Contribution of CXCL12 secretion to invasion of breast cancer cells. Breast Cancer Res. 14, 1-14 (2012).
56. Grundker, C. et al. Inhibition of SDF-1/CXCR4-induced epithelialmesenchymal transition by kisspeptin-10. Breast Cancer Res. Treat. 152, 41-50 (2015)

57. Kong, L. et al. Overexpression of SDF-1 activates the NF-kappaB pathway to induce epithelial to mesenchymal transition and cancer stem cell-like phenotypes of breast cancer cells. Int. J. Oncol. 48, 1085-1094 (2016).

58. Martinez-Ordonez, A. et al. Breast cancer metastasis to liver and lung is facilitated by Pit-1-CXCL12-CXCR4 axis. Oncogene 37, 1430-1444 (2018).

59. Felipe Lima, J., Nofech-Mozes, S., Bayani, J. \& Bartlett, J. M. EMT in Breast Carcinoma-A Review. J. Clin. Med. 5, E5 (2016).

60. Fedele, M., Cerchia, L. \& Chiappetta, G. The epithelial-to-mesenchymal transition in breast cancer: focus on basal-like carcinomas. Cancers (Basel) $\mathbf{9}$, E134 (2017).

61. Ohlund, D., Elyada, E. \& Tuveson, D. Fibroblast heterogeneity in the cancer wound. J. Exp. Med. 211, 1503-1523 (2014).

62. Kuzet, S. E. \& Gaggioli, C. Fibroblast activation in cancer: when seed fertilizes soil. Cell Tissue Res. 365, 607-619 (2016).

63. Takai, K., Le, A., Weaver, V. M. \& Werb, Z. Targeting the cancer-associated fibroblasts as a treatment in triple-negative breast cancer. Oncotarget 7, 82889-82901 (2016)

64. Albrengues, J. et al. LIF mediates proinvasive activation of stromal fibroblasts in cancer. Cell Rep. 7, 1664-1678 (2014).

65. Bonan, S. et al. Membrane-bound ICAM-1 contributes to the onset of proinvasive tumor stroma by controlling acto-myosin contractility in carcinoma-associated fibroblasts. Oncotarget 8, 1304-1320 (2016).

66. Ranganathan, P., Weaver, K. L. \& Capobianco, A. J. Notch signalling in solid tumours: a little bit of everything but not all the time. Nat. Rev. Cancer 11, 338-351 (2011).

67. Lamy, M. et al. Notch-out for breast cancer therapies. New Biotechnol. 39, 215-221 (2017).

68. Shen, Q. et al. Notch shapes the innate immunophenotype in breast cancer. Cancer Discov. 7, 1320-1335 (2017).

69. Del Valle, P. R. et al. Transcriptional profile of fibroblasts obtained from the primary site, lymph node and bone marrow of breast cancer patients. Genet. Mol. Biol. 37, 480-489 (2014).

70. Rodda, L. B. et al. Single-cell RNA sequencing of lymph node stromal cells reveals niche-associated heterogeneity. Immunity 48, 1014-1028.e1016 (2018)

\section{Acknowledgements}

We thank Dr. V. Mieulet and G. Gentric for critical reading of the manuscript and all members of F.M.-G.'s laboratory for fruitful discussions. We are grateful to Dr. V. Becette, M. Toutain and A. Duchenne for providing access to some tumor samples. We thank Z. Maciorowski, S. Grondin and A. Viguier from the Flow cytometry platform at Institut Curie (IC). We also thank R. Leclere, E. Martel and A. Nicolas from the experimental pathology platform (IC); S. Baulande, S.Lameiras, V. Raynal, M. Bohec and P. Legoix from the NGS platform (IC) for their help and advice. We acknowledge the Cell and Tissue Imaging (PICT-IBiSA) and Nikon Imaging Centre (IC) member of the French National Research Infrastructure France-BioImaging (ANR10-INBS-04). In particular, we thank O. Renaud and O. Leroy (IC) for technical assistance with microscopy and IMARIS software. We thank K. Laud for her help with spheroids experiments F.P. was supported by funding from Ecole des Mines de Paris and from the Foundation ARC (Association pour la Recherche sur le Cancer). F.M.-G. and C.B. are supported by Institut National de la Santé et de la Recherche Médicale (Inserm). Y.K. was funded by the Foundation pour la Recherche Médicale (FRM), SIRIC (INCa-DGOS-4654) and INCa (INCa-DGOS-9963). M.C.P. was supported by FRM (ING20160435436) and Inserm (EQ2016-01). The experimental work was supported by grants from Ligue Nationale Contre le Cancer (Labelisation), Inserm, Institut Curie (PIC TME/T-MEGA and PIC3i CAFi), ICGex (ANR-10-EQPX-03), SIRIC (INCa-DGOS-4654) and INCa (INCa-DGOS-9963, INCa-2016-166, INCa-11692, INCa-DGOS-Inserm-12554) and ARCS. F.M.-G. acknowledges both the Association Le cancer du sein, Parlons-en and the Simone and Cino del Duca Foundation for attribution of their "Grand Prix". F.M.-G. is very grateful to her funders for providing support these last years.

\section{Author contributions}

F.M.-G. conceived the project, and together with F.P. designed the experiments. F.P., B.B. I.M., A.C., A.-M.G. performed the experiments. F.P. and Y.K. performed bioinformatic and statistical analyses of the data. M.C.P. and S.D. designed the tumor-on-chip system used for video-microscopy and F.M.-M. performed and analyzed these experiments. I.B. and P.S. designed TFM experiments and I.B. analyzed these experiments. Y.A., J.B. and D.V. designed the SHG and inverted Transwell assays and helped with the microscopy acquisition. C.B., L.F. and A.V.-S. provided human samples analyzed in the study. F.M.-G. and F.P. wrote the manuscript with input from all authors. F.M.-G. supervised the entire project.

\section{Competing interests}

The authors declare no competing interests. 


\section{Additional information}

Supplementary information is available for this paper at https://doi.org/10.1038/s41467019-14134-w.

Correspondence and requests for materials should be addressed to F.M.-G.

Peer review information Nature Communications thanks Armando del Río Hernández and the other anonymous reviewer(s) for their contribution to the peer review of this work. Peer reviewer reports are available.

Reprints and permission information is available at http://www.nature.com/reprints

Publisher's note Springer Nature remains neutral with regard to jurisdictional claims in published maps and institutional affiliations. (c) (i) Open Access This article is licensed under a Creative Commons Attribution 4.0 International License, which permits use, sharing, adaptation, distribution and reproduction in any medium or format, as long as you give appropriate credit to the original author(s) and the source, provide a link to the Creative Commons license, and indicate if changes were made. The images or other third party material in this article are included in the article's Creative Commons license, unless indicated otherwise in a credit line to the material. If material is not included in the article's Creative Commons license and your intended use is not permitted by statutory regulation or exceeds the permitted use, you will need to obtain permission directly from the copyright holder. To view a copy of this license, visit http://creativecommons.org/ licenses/by/4.0/.

(C) The Author(s) 2020 